\title{
INTERPOLATION PROPERTIES OF GENERALIZED PERFECT SPLINES AND THE SOLUTIONS OF CERTAIN EXTREMAL PROBLEMS. I
}

BY

\author{
SAMUEL KARLIN( $\left.{ }^{1}\right)$
}

\begin{abstract}
The existence of generalized perfect splines satisfying certain interpolation and/or moment conditions are established. In particular, the existence of ordinary perfect splines obeying boundary and interpolation conditions is demonstrated; precise criteria for the uniqueness of such interpolatory perfect splines are indicated. These are shown to solve a host of variational problems in certain Sobolev spaces.
\end{abstract}

1. Introduction. A perfect spline of degree $n(n \geqslant 1)$ with $r$ knots on [0, 1] is a function of the form

$$
P(x)=c\left[x^{n}+2 \sum_{i=1}^{r}(-1)^{i}\left(x-\xi_{i}\right)_{+}^{n}\right]+\sum_{j=0}^{n-1} a_{j} x^{j}
$$

where $c, a_{0}, a_{1}, \cdots, a_{n-1}$ are real constants and the knots $\left\{\xi_{i}\right\}$ obey the constraints $0<\xi_{1}<\xi_{2}<\cdots<\xi_{r}<1$. The perfect spline exhibits the property that its $n$th derivative though changing sign at each knot maintains a constant absolute value, in this case $|c| n !$. Glaeser appears to have been the first to emphasize the class of perfect splines. These functions are fundamental to the solution of several types of extremal problems in certain Sobolev spaces, see e.g. Glaeser [2], Tihomirov [12], Schoenberg [10], Schoenberg and Cavaretta [11], Karlin [5]. Schumaker and others, call these bang-bang splines attributable to the constant absolute value of $P^{(n)}(x)$, and the property that this derivative changes sign at the knots. Considerable evidence points to the fact that perfect splines feature prominently in the solution of certain optimization problems occurring in statistics, control theory and certain facets of numerical analysis.

We cite two typical problems:

1. Let $W^{(n)}[0,1]$ be the Sobolev space comprised of all functions $f$ defined on $[0,1]$ where $f^{(n-1)}$ is absolutely continuous and the maximum norm of its $n$th derivative $\left\|f^{(n)}\right\|$ is finite. Let $x_{1} \leqslant x_{2} \leqslant \cdots \leqslant x_{n+r+1}$ be $n+$

Received by the editors February 13, 1974.

AMS(MOS) subject classifications (1970). Primary 41 A15; Secondary 46E35.

(1) Research supported in part by ONR Contract N00014-67-A-0112-0015 at Stanford. 
$r+1$ prescribed points in $[0,1]$ involving no coincident block exceeding $n$ points and let $\left\{\alpha_{i}\right\}_{1}^{n+r+1}$ be given real numbers (not all zero). Consider the subset $J[\mathbf{x}, \alpha] \subset W_{\infty}^{(n)}$ consisting of all $f \in W_{\infty}^{(n)}$ interpolating the data $D=\left\{\alpha_{i}\right\}$ at $\mathrm{X}=\left\{x_{i}\right\}$ i.e. $f \in J[\mathbf{x}, \alpha]$ entails that $f \in W_{\infty}^{(n)}$ satisfies

$$
f\left(x_{i}\right)=\alpha_{i}, \quad i=1,2, \cdots, n+r+1 .
$$

(When coincident $x_{i}$ occur, then the interpolation conditions are interpreted as interpolation for the appropriate successive higher derivatives of that $x$ value.) A problem of interest in approximation theory and elsewhere is to characterize the minimizing function (or functions) yielding

$$
\min _{f \in J[\mathbf{x}, \alpha]}\left\|f^{(n)}\right\|_{\infty}
$$

The existence of a minimum in (1.3) can be established by functional analysis techniques. A characterization of a minimum in (1.3) can also be obtained by direct methods (see Karlin and Micchelli [1975]). The methods of this paper proceed via a general covering theorem of deeper content and bearing widely on other applications. One of the applications will establish that the minimum (1.3) is attained by a perfect spline (usually not necessarily uniquely when $r>n$ ) involving at most $r$ knots. The special case where $r=n-1$ and $x_{1}=x_{2}=\cdots=$ $x_{n}=0, x_{n+1}=x_{n+2}=\cdots=x_{2 n}=1$, was investigated by Glaeser who exploited a special form of Taylor's expansion with a remainder formula combined with some functional analysis and convexity arguments.

2. Consider the collection $U(\mathrm{c})$ of all $W_{\infty}^{(n)}[0,1]$ functions satisfying the constraints

$$
\int_{0}^{1} x^{i-1} f(x) d x=c_{i}, \quad i=1,2, \cdots, t+1 \quad(t \geqslant n)
$$

for given real constants $c_{i}$. The problem is to determine $f \in U(c)$ achieving

$$
\min _{f \in U(\mathrm{c})}\left\|f^{(n)}\right\|_{\infty} \text {. }
$$

We establish in $\S 7$ that the unique minimizing $f$ is a perfect spline of degree $n+t+1$ involving $t-n$ knots. Actually the special problem of (1.5a) can be reduced to that of problem 1 .

The above problems suggest a more general formulation encompassing both cases. Let $\mu_{1}, \mu_{2}, \cdots, \mu_{t+1}$ be $t+1$ signed measures of bounded variation defined on $[0,1]$. Consider all $f \in W_{\infty}^{(n)}$ fulfilling the conditions

$$
\int_{0}^{1} f(x) d \mu_{i}(x)=c_{i}, \quad i=1,2, \cdots, t+1, t \geqslant n .
$$


Call this class $W(\mu, \mathrm{c})$. Determine $f \in W(\mu, \mathrm{c})$ attaining

$$
\min _{f \in W(\mu, \mathrm{c})}\left\|f^{(n)}\right\|_{\infty}
$$

If the measures $\left\{\mu_{i}\right\}$ form a totally positive sequence as delimited in $\S 7$ then the last minimum is achieved by a perfect spline exhibiting at most $t-n$ knots.

An extended version of the problem in (1.3) involves replacing the $n$th derivative operator by an $n$th order differential operator of the form

$$
D^{n} f=\left(\frac{d}{d x} \frac{1}{w_{n}(x)} \frac{d}{d x} \frac{1}{w_{n-1}(x)} \cdots \frac{d}{d x} \frac{1}{w_{1}(x)}\right) f(x),
$$

where $w_{i}(x) \in C^{n}[0,1]$ and $w_{i}(x)>0, i=1,2, \cdots, n$, on $[0,1]$.

A key to the characterization of the solution of the foregoing variational problems (especially (1.3)) is the following more basic interpolation theorem, deduced as a consequence of the more general covering theorem stated later in this introduction.

THEOREM 1. Let $\left\{x_{i}\right\}_{1}^{n+r+1}(n \geqslant 1, r \geqslant 0)$ be prescribed with $0 \leqslant x_{1} \leqslant$ $x_{2} \leqslant \cdots \leqslant x_{n+r+1} \leqslant 1$ involving no coincident block exceeding $n$ points. Let $\left\{\alpha_{i}\right\}_{1}^{n+r+1}$ be any given real data satisfying $\sum_{i=1}^{n+r+1} \alpha_{i}^{2}>0$. Then there exists a perfect spline $P(x)$ (not necessarily unique) of the form (1.1) with at most $r$ knots such that $P\left(x_{i}\right)=\alpha_{i}, i=1,2, \cdots, n+r+1$.

(Criteria for uniqueness are elaborated in §6.)

The existence part of Theorem 1 (with emphasis on the property of "at most $r$ knots") appears to be nonelementary. Our analysis uses some topological techniques and further properties akin to the total positivity nature of the kernel $K(x, \xi)=(x-\xi)_{+}^{n}$.

As mentioned earlier, the special case $x_{1}=x_{2}=\cdots=x_{n}=0, x_{n+1}=$ $x_{n+2}=\cdots=x_{2 n}=1$ is considerably easier. In this case the solution is unique. An explicit determination of the interpolating perfect spline for the interpolation conditions $P(0)=P^{\prime}(0)=\cdots=P^{(n-1)}(0)=0, P(1)=1, P^{\prime}(1)=\cdots=$ $P^{(n-1)}(1)=0$ was given by Louboutin [9] where the knots can be identified as "essentially" the zeros of the classical Tchebycheff polynomial of the second kind.

An extension of Theorem 1 allowing a more general prescription of interpolating conditions at the boundary is the content of the next theorem.

THEOREM 2. Let $0 \leqslant i_{1}<i_{2}<\cdots<i_{p} \leqslant n-1$ and $0 \leqslant j_{1}<j_{2}<$ $\cdots<j_{q} \leqslant n-1$ be $p$ and $q$ prescribed indices associated with the endpoints 0 and 1 respectively. Let $0<x_{1} \leqslant x_{2} \leqslant \cdots \leqslant x_{k}<1$ be given with no co- 
incident block exceeding $n$ points. Suppose $p+k+q=n+r+1, n \geqslant 1$. Let $\left\{\gamma_{i}\right\}_{1}^{p},\left\{\alpha_{i}\right\}_{1}^{k}$ and $\left\{\delta_{i}\right\}_{1}^{q}$ be given real values not all zero. Then there exists a perfect spline $P(x)$ of the form (1.1) with at most $r$ knots satisfying

$$
\begin{aligned}
P^{\left(i_{\nu}\right)}(0) & =\gamma_{\nu}, & v & =1,2, \cdots, p, \\
P\left(x_{i}\right) & =\alpha_{i}, & i & =1,2, \cdots, k, \\
P^{\left(j_{\mu}\right)}(1) & =\delta_{\mu}, & \mu & =1,2, \cdots, q,
\end{aligned}
$$

provided the interpolation requirements in (1.6) obey the Pólya condition i.e., the cumulative number of stipulations on $P(x)$ and up to the mth derivative exceeds $m, m=0,1, \cdots, n$.

For the relevance of the Pólya conditions to these kinds of considerations consult Karlin and Karon [6].

A further generalization of Theorem 2 involving boundary conditions of the type usually coupled to differential operators (cf. [4]) is also forthcoming. We will not describe the corresponding results.

A consequence of Theorem 1 is:

THEOREM 3. The perfect spline affirmed in Theorem 1 provides a minimum in (1.3).

The lack of uniqueness in Theorems $1-3$ prominent for $r>n$ is disconcerting and is mainly attributable to the fact that the system of functions

$$
u_{k}(\xi)=\left(x_{k}-\xi\right)_{+}^{n} \quad(0 \leqslant \xi \leqslant 1) k=1,2, \cdots, r,
$$

constitutes a weak Tchebycheff system (T. S.) rather than a bona fide Tchebycheff system (T. S.). (A T. S. is characterized by the property that every nontrivial real linear combination $v(x)=\sum_{i=1}^{n} a_{i} v_{i}(x)$ vanishes at most $n-1$ times and if its number of zeros $Z(v ;[0,1])=n-1, v(x)$ necessarily changes sign at every zero interior to $[0,1]$.)

The results of Theorems 1, 2 and Theorem 7.1 later, and other applications will be deduced executing suitable adaptations on Theorem 4 . We emphasize here that this theorem encompasses our main global theorem and is more far reaching than merely for the context of perfect splines.

THEOREM 4. Let $\left\{\mu_{i}(x)\right\}_{1}^{n+r+1}$ be a family of functions such that $\left\{v_{i}(x)=d u_{i}(x) / d x\right\}_{1}^{n+r+1}$ are piecewise continuous. Let $\left\{\alpha_{i}\right\}_{1}^{n+r+1}$ be real data with $\Sigma \alpha_{i}^{2}>0$. Suppose $\left\|A_{i j}\right\|_{i=1, j=1}^{n+r+1, n}$ is a matrix with the property that for any $0<\eta_{1}<\eta_{2}<\cdots<\eta_{s}<1$ and $s \leqslant r+1$, the determinant 


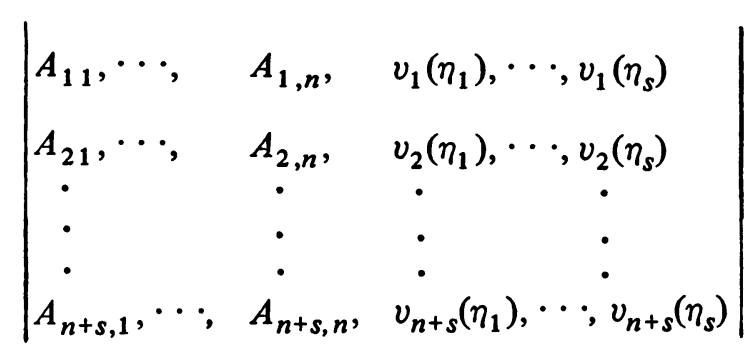

keeps a single strict sign. Then, there exists $a$ set of real values $\left\{a_{j}\right\}_{j=1}^{n}$ and $c$, $\sum_{j=1}^{n} a_{j}^{2}+c^{2}>0$ and $0<\xi_{1}<\xi_{2}<\cdots<\xi_{s}<1, s \leqslant r$ (usually, we have $s=r$ ) satisfying

$$
\begin{array}{r}
\sum_{j=1}^{n} A_{i j} a_{j}+c\left[u_{i}(1)+2(-1)^{s+1} \sum_{j=1}^{s}(-1)^{j} u_{i}\left(\xi_{j}\right)+(-1)^{s+1} u_{i}(0)\right]=\alpha_{i}, \\
i=1,2, \cdots, n+r+1 .
\end{array}
$$

For any data $\left\{\alpha_{i}\right\}_{1}^{n+r+1}$ not in the range of the matrix $A=\left\|A_{i k}\right\|$, the solution of (1.8) entailing the minimal number of knots (we use the terminology knots referring to the $\left\{\xi_{i}\right\}$ even in the present general context) is uniquely determined. For example, where the data $\left\{\alpha_{i}\right\}_{1}^{n+r+1}$ consists of all zero data but for one component, then the system (1.8) is solved uniquely and involves $r$ knots.

REMARK. The uniqueness conditions applicable to Theorem 1 are considerably weaker than the criteria set forth in Theorem 4 . (See $\$ 6$ for a complete discussion.)

Note in (1.8) the appearance of the term $u_{i}(1)$. For the special examples of Theorems 1 and 2 we have $u_{i}(1)=0$ and therefore the contribution of this term is missing.

As mentioned above, Theorems 1 and 2 are not immediate consequences of Theorem 3 since the collection of functions $u_{i}(\xi)=\left(x_{i}-\xi\right)_{+}^{n}, i=1,2, \cdots, r$, in conjunction with the matrix $\left\|A_{i k}\right\|=\left\|x_{i}^{k-1}\right\|$ and their derivatives $v_{i}(\xi)=$ $-n\left(x_{i}-\xi\right)_{+}^{n-1}$ do not strictly obey the requirement of (1.7). Actually, no sign change is possible for the determinants (1.7) in this case but they can be zero. To deduce Theorems 1 and 2 we implement a standard smoothening of $u_{i}(\xi)$ so that Theorem 4 becomes applicable ( $\S 4)$. A limiting process is then performed leading to the conclusions of Theorem 1 but without the uniqueness statement persisting even where $c \neq 0$.

Further topological arguments involving mainly applications of the Brouwer fixed point theorem with reliance on the result of Theorem 4 will enable us to establish the existence of the two special perfect splines described in the next theorem.

THEOREM 5. Let $g(x)$ and $h(x)$ be continuous positive and nonpositive, 
respectively, on $[0,1]$. Let $n$ and $r$ be given and suppose that $n+r=k$. Then, there exists two special perfect splines $\widetilde{P}(x)$ and $\underset{\sim}{P}(x)$ with $r$ knots, each oscillating maximally between $g(x)$ and $h(x)$ in the following sense:

(i) $h(x) \leqslant \widetilde{P}(x) \leqslant g(x)$ on $[0,1]$;

(ii) there exists $k+1$ points $0 \leqslant z_{1}<\cdots<z_{k+1} \leqslant 1$ such that

$$
\widetilde{P}\left(z_{i}\right)= \begin{cases}g\left(z_{i}\right), & i \text { odd }, \\ h\left(z_{i}\right), & i \text { even } .\end{cases}
$$

Similarly, the perfect spline $\underset{\sim}{P}(x)$ is characterized by the conditions (i) as above and (ii'), arising from (ii) by interchanging the functions $g(x)$ and $h(x)$.

Several important corollaries ensue out of Theorem 5. We highlight one of them.

COROLlary 1. Let $Q$ be any positive polynomial on $[0,1]$ of degree $\leqslant n-1$. Let $r$ be a given positive integer. Then the unique representation

$$
Q(x)=\tilde{P}(x)+\underset{\sim}{P}(x) \text { for all } x
$$

prevails where $\widetilde{P}$ and $\underset{\sim}{P}$ are the maximally oscillating splines of Theorem 5 associated with $g(x)=Q(x)$ and $h(x) \equiv 0$.

Specializing Theorem 5 to the case of $h(x) \equiv-1$ and $g(x) \equiv 1$ we obtain

THEOREM 6. For given $n$ and $r$ there exists a unique perfect spline $P_{n, r}(x)$ of degree $n$ with $r$ knots oscillating maximally over the interval $[0,1]$ between 1 and -1 in the sense of Theorem 5 and normalized by the condition $P_{n, r}(0)=1$. It follows that $P_{n, r}(1)=(-1)^{n+r}$.

The construction of the special $P_{n, r}$ can be achieved without reliance on the full force of Theorems 1 and 5 by appropriate application of the Brouwer fixed point theorem. The case of Theorem 6 was announced by Schoenberg and Cavaretta [11]. The function $P_{n, r}$ also features prominently in the work of Tihomirov [12].

Designate $\rho_{n, r}$ as the constant value $\left\|P_{n, r}^{(n)}\right\|_{\infty}=\rho_{n, r}^{-1}(1)=\left|P_{n, r}^{(n)}(1)\right|$. It is usually more convenient to normalize the $n$th derivative to 1 . Accordingly, we form

$$
\begin{aligned}
& Q_{n, r}(x)=\rho_{n, r} P_{n, r}(x) \text { so that } \\
& \left\|Q_{n, r}\right\|_{\infty}=\rho_{n, r}=Q_{n, r}(0)=(-1)^{n+r} Q_{n, r}(1), \text { and } \\
& \left\|Q_{n, r}^{(n)}\right\|_{\infty}=1 .
\end{aligned}
$$


It can be proved that $\rho_{n, 0}>\rho_{n, 1}>\rho_{n, 2}>\cdots>\rho_{n, r} \rightarrow 0$ as $r \rightarrow \infty$.

A more basic version of Theorem 6 is stated below whose proof falls back on the interpolation property enunciated in Theorem 4 and some further topological analysis.

THEOREM 7. Let $\rho$ be prescribed satisfying $0<\rho<\infty$. There exists a unique perfect spline $Q(x ; \rho)$ of degree $n$ satisfying $\left|d Q^{(n)}(x ; \rho) / d x^{n}\right| \equiv 1$, $|Q(x ; \rho)| \leqslant \rho$ on $[0,1]$ and $Q(0 ; \rho)=\rho$. Moreover, if $\rho_{n, r-1}>\rho>\rho_{n, r}$ $\left(\rho_{n,-1}=+\infty\right.$ by convention), $Q(x ; \rho)$ involves exactly $r$ knots and oscillates $n+r$ times between $\rho$ and $-\rho$, as $x$ traverses [0,1]. Also, $Q(x ; \rho)$ vanishes $n+r$ times on $[0, \infty)$, displaying at most one zero outside $[1, \infty)$. When, $\rho=\rho_{n, r}$, of course, $Q(x, \rho)=Q_{n, r}(x)$.

The perfect splines $Q(x ; \rho)$ enjoy several remarkable optimum properties. We highlight three typical situations.

(a) Consider the class $\mathcal{C}(\rho)$ of all $f \in W_{\infty}^{(n)}$ defined on $[0,1]$ obeying the restrictions

$$
\left\|f^{(n)}\right\|_{\infty} \leqslant 1 \quad \text { and }\|f\| \leqslant \rho, \quad \rho>0
$$

Then for any $1 \leqslant \nu \leqslant n-1, \max _{f \in C(\rho)}\left|f^{(\nu)}(0)\right|=\left|Q^{(\nu)}(0 ; \rho)\right|$.

(b) Let $0<x^{*}<1$. Consider the class $C\left(\rho ; x^{*}\right)$ of $f$ in $C(\rho)$ with the further property that $f\left(x^{*}\right)=Q\left(x^{*}, \rho\right)$. Suppose $Q^{\prime}\left(x^{*}, \rho\right)>0$. (If negative, replace $\max$ in $(1.12)$ by $\min$.) We have

$$
\max _{f \in C\left(\rho ; x^{*}\right)} f^{\prime}\left(x^{*}\right)=Q^{\prime}\left(x^{*} ; \rho\right) .
$$

(c) Let $n+r=2 m$. Suppose $f \in W_{\infty}^{(n)}$ on $[0, b], b>1$, satisfies the constraints $\left\|f^{(n)}\right\|_{\infty} \leqslant 1$ on $[0, b]$ but $\|f\| \leqslant \rho_{n, r}$ on $[0,1]$. Call this class of functions $C_{n, r}[0, b]$. Then, we have

$$
\max _{f \in C_{n, r}[0, b]} f(b)=\left|Q_{n, r}(b)\right| .
$$

We now set forth the concept of a perfect spline associated with certain $n$th order differential operators.

Let $\left\{w_{i}(x)\right\}_{1}^{n}$ be positive and of class $C^{n}$ on $[0,1]$. Consider the $n$th order differential operator $L_{n} v=D_{n} D_{n-1} \cdots D_{1} v$ composed from the first order differential operators

$$
\left(D_{i} u\right)(x)=\frac{d}{d x}\left(\frac{1}{w_{i}(x)} u(x)\right), \quad i=1,2, \cdots, n,
$$

acting on $u \in C^{n}[0,1]$. The solutions of $L_{n} u=0$ analogous to the powers $\left\{x^{i}\right\}_{0}^{n-1}$ are $\left\{u_{i}(x)\right\}_{0}^{n-1}$, where $u_{0}(x)=w_{1}(x)$, 


$$
\begin{array}{r}
u_{i}(x)=w_{1}(x) \int_{0}^{x} w_{2}\left(t_{1}\right) \int_{0}^{t_{1}} w_{3}\left(t_{2}\right) \cdots \int_{0}^{t_{i-1}} w_{i+1}\left(t_{i}\right) d t_{i} d t_{i-1} \cdots d t_{1}, \\
i=1,2, \cdots, n-1 .
\end{array}
$$

We let $\phi_{n}(x ; \xi)$ be the fundamental solution of $L_{n} \phi=0$.

A "generalized perfect spline" corresponding to the differential operator $L_{n}=D^{n}$ (we have changed notation here) is an expression of the form

$$
c\left[u_{n}(x)+2 \sum_{i=1}^{r}(-1)^{i} \phi\left(x, \xi_{i}\right)\right]+\sum_{i=0}^{n-1} a_{i} u_{i}(x)
$$

where $u_{n}(x)$ satisfies $D^{n} u_{n}(x) \equiv 1$ subject to the initial conditions $u_{n}(0)=$ $D^{1} u_{n}(0)=\cdots=D^{n-1} u_{n}(0)=0$, and $\phi(x, \xi)$ is the fundamental solution of the operator $L_{n+1} f=d\left(D^{n} f\right) / d x$. The analogues of Theorems 1 and 2 persist for generalized perfect splines. The Sobolev space $W_{\infty}\left(D^{n}\right), L_{n}=D^{n}$, is determined as consisting of all functions such that $D^{n} f$ is defined a.e. and $\left\|D^{n} f\right\|_{\infty}$ is finite. Theorem 3 admits the following version for $W_{\infty}\left(L_{n}\right)$.

THEOREM $3^{\prime}$. Let $J\left(\mathrm{x}, \alpha, D^{n}\right)$ consist of all $f \in W_{\infty}\left(D^{n}\right)$ interpolating the data $\left\{\alpha_{i}\right\}$ at $\left\{x_{i}\right\}$. Then $\min _{f \in J}\left\|D^{n} f\right\|_{\infty}$ is achieved by a "generalized perfect spline" of the form (1.13) interpolating the given data.

We conclude this introduction by indicating the organization of the paper.

The proof of Theorem 4 is relegated to $\$ 3$ while in $\$ 2$ a number of ancillary lemmas are developed. The results of $\$ 2$ enable us to verify the global one to one character of a natural mapping associated with the equations (1.8). The proof of Theorem 4 utilizes a variety of topological analyses.

$\S 4$ is devoted to the proof of Theorem 1 . The problem of uniqueness of the interpolating perfect spline, its scope, extent and limitations, is discussed in $\S 6$. A variety of auxiliary properties, possessing independent interest on perfect splines are set forth in $\S 5$. For example, we locate the knots relative to the interpolating points for certain cases.

$\S \S 7$ and 8 present the solutions of the extremal problems of (1.5a) and (1.5b). Here, some further variational problems and representation theorems connected with perfect splines, not described in this introductory section, are settled. The elaboration of the proofs of Theorems 5-7 and further ramifications and applications of the theory of perfect splines are deferred to a separate publication.

After completing this paper in 1972, the work of Fisher and Jerome [1] was brought to our attention. They achieve a local result in characterizing the solutions of the variational problems of the kind (1.3) under more general interpolating conditions. The solutions involve perfect splines subject to the gross bound 
of at most $n$ knots between each two interpolating points. Therefore, for $n+r+1$ interpolating points, their solution could conceivably have $n(n+r+1)$ knots. Our results are global in character providing the exact bound of $r$ knots in the case of $n+r+1$ Hermite interpolating data. Moreover, the covering Theorem 4 can be used for other applications and transcends the context of interpolation by polynomials.

Added in proof. We have achieved (in collaboration with C. Michelle) a variational approach to Theorem 4 .

2. Measures induced by knots of perfect splines and orthoganility properties. $\S \S 3$ and 4 are devoted to proofs of the main interpolation Theorems 1 and 4 , (stated in \$1) and several extensions. The relevance and need for the following lemmas will become manifest during the later developments. The reader not wishing to be encumbered by technical details can pass directly to $\S 3$ and return later here.

LEMMA 2.1. Consider two collections of ordered numbers

$$
1>\alpha_{1}>\alpha_{2}>\cdots>\alpha_{2 m-1}>0,1>\beta_{1}>\beta_{2}>\cdots>\beta_{l}>0 .
$$

(Note that the $\alpha$ 's and $\beta$ 's appear in decreasing magnitude.)

Regroup the numbers of (2.1) into the two sets $S_{1}$ and $S_{2}$ as follows:

$$
\begin{aligned}
& S_{1}=\left\{1, \alpha_{2}, \alpha_{4}, \alpha_{6}, \cdots, \alpha_{2 m-2}, \beta_{1}, \beta_{3}, \cdots, \beta_{l^{*}}, 0\right\} \text {, } \\
& l^{*}=\left\{\begin{array}{l}
l \text { if } l \text { is odd, } \\
l-1 \text { if } l \text { is even. }
\end{array}\right. \\
& S_{2}=\left\{\alpha_{1}, \alpha_{3}, \alpha_{5}, \cdots, \alpha_{2 m-1}, \beta_{2}, \beta_{4}, \beta_{6}, \cdots, \beta_{l}\right\} \text {, } \\
& \hat{l}=\left\{\begin{array}{l}
l-1 \text { if } l \text { is odd }, \\
l \text { if } l \text { is even. }
\end{array}\right.
\end{aligned}
$$

Let $c \geqslant 1$. Construct the increasing step function $F(x)$ whose points of increase are those of $S_{1}$ with upward jumps of magnitude $2 c$ and 2 at the points $\alpha_{2 i}$ and $\beta_{2 i+1}$, respectively, the jump $c-1$ at 1 , and jumps $c-1$ at 0 if $l$ is odd and $c+1$ if $l$ is even. When an $\alpha$ point coincides with a $\beta$, then cumulate the jumps.

Next construct the increasing step function $G(x)$ associated with $S_{2}$ with jumps of size $2 c$ at $\alpha_{2 i+1}$ and magnitude 2 at $\beta_{2 i}$. Then $F(x)-G(x)$ changes sign at most $2 m-1$ times.

Proof. Note that $F\left(0^{-}\right)=G\left(0^{-}\right)=0$, and $F(1)=G(1)=2 c m+2[l / 2]$. Also 


$$
F(0+)= \begin{cases}c-1 & \text { if } l \text { is odd } \\ c+1 & \text { if } l \text { is even }\end{cases}
$$

and $F(1)-F(1-)=c-1$ accordingly.

As $x$ decreases from 1 towards $\alpha_{1}, G(x)-F(x)$ takes the values $c-1$ $c+1$, changing from one to the other as successive $\beta_{i}$ points are encountered (if they are encountered at all). The relevant fact is that $G-F$ is nonnegative after $\alpha_{1}$. At $\alpha_{1}, G$ jumps by $2 c$ so that $G-F$ drops down to $-(c+1)$ or $-(c-1)$ just before $\alpha_{1}$. As $x$ proceeds downward toward $\alpha_{2}, G-F$ alternates between these two latter values, the jumps occurring at the $\beta$ 's (if there are any in $\left(\alpha_{2}\right.$, $\left.\alpha_{1}\right)$ ). Thus, $G-F$ is nonpositive on $\left(\alpha_{2}, \alpha_{1}\right)$. At $\alpha_{2}$ - another sign change occurs back to the value $c+1$ or $c-1$. In summary, $G-F$ strictly changes sign possibly at the $2 m-1$ points $\alpha_{1}, \alpha_{2}, \cdots, \alpha_{2 m-1}$ and nowhere else. This clearly remains valid, if any of the $\beta$ 's coincide with any of the $\alpha$ 's (by a standard continuity argument). The proof is complete.

Lemma 2.2. Let the sets $\left\{\alpha_{i}\right\}$ and $\left\{\beta_{i}\right\}$ be given as in Lemma 2.1. Here form the sets

$$
\begin{aligned}
& \tilde{S}_{1}=\left\{1, \alpha_{2}, \alpha_{4}, \cdots, \alpha_{2 m-2}, \beta_{2}, \beta_{4}, \cdots, \beta_{\tau}, 0\right\}, \\
& \tilde{S}_{2}=\left\{\alpha_{1}, \alpha_{3}, \cdots, \alpha_{2 m-1}, \beta_{1}, \beta_{3}, \cdots, \beta_{l^{*}}\right\} .
\end{aligned}
$$

(Note that now $\tilde{S}_{1}$ includes the points $\beta_{2 i}$ instead of $\beta_{2 i-1}$.) Let $\tilde{F}(x)$ be constructed with upward jumps of amount $2 c$ at $\alpha_{2 i}$ and of magnitude $c+1$ $(c-1)$ at 0 if $l$ is odd (even) 2 at $\beta_{2 i}$ and a jump of magnitude $c+1$ at 1 . Let $\widetilde{G}(x)$ be the increasing step function with jumps of magnitude $2 c$ at $\alpha_{2 i-1}$ and 2 at $\beta_{2 i-1}$. Then $F(x)-G(x)$ changes sign at most $2 m-1$ times.

The proof paraphrases that of Lemma 2.1.

We record now the versions of Lemmas 2.1 and 2.2 when there appears an even set of points.

LEMmA 2.3. Let $1>\alpha_{1}>\alpha_{2}>\cdots>\alpha_{2 m}>0$ and $1>\beta_{1}>\beta_{2}>$ $\cdots>\beta_{l}>0$. Consider the sets

$$
\begin{aligned}
& S_{1}=\left\{1, \alpha_{2}, \alpha_{4}, \cdots, \alpha_{2 m}, \beta_{1}, \beta_{3}, \cdots, \beta_{l^{*}}\right\}, \\
& S_{2}=\left\{\alpha_{1}, \alpha_{3}, \cdots, \alpha_{2 m-1}, \beta_{2}, \beta_{4}, \cdots, \beta_{\tau}, 0\right\} .
\end{aligned}
$$

Let $c \geqslant 1$. Form the increasing step functions:

$F(x)$ jumps $2 c$ at $\alpha_{2 i}, 2$ at $\beta_{2 i-1}$ and $c-1$ at 1 . 
$G(x)$ jumps $2 c$ at $\alpha_{2 i-1}, 2$ at $\beta_{2 i}$ and $c-1$ at 0 if $l=$ even, $c+1$ at 0 if $l=o d d$.

Then $F(x)-G(x)$ changes sign at most $2 m$ times.

Lemma 2.4. Consider $\left\{\alpha_{i}\right\}$ and $\left\{\beta_{j}\right\}$ as in Lemma 2.3. Let $c \geqslant 1$ and form

$$
\begin{aligned}
& \tilde{S}_{1}=\left\{1, \alpha_{2}, \alpha_{4}, \cdots, \alpha_{2 m}, \beta_{2}, \beta_{4}, \cdots, \beta_{\tau}\right\}, \\
& \tilde{S}_{2}=\left\{\alpha_{1}, \alpha_{3}, \cdots, \alpha_{2 m-1}, \beta_{1}, \beta_{3}, \cdots, \beta_{l}, 0\right\} .
\end{aligned}
$$

Construct $\widetilde{F}(x)$ analogous to $F(x)$ but having a jump of $c+1$ at $1, \widetilde{G}(x)$ is correspondingly changed from $G(x)$ in that it exhibits a jump of magnitude $c+1$ or $c-1$ at 0 (according as $l$ is even or odd). Then, $\widetilde{F}(x)-\widetilde{G}(x)$ exhibits at most $2 m$ sign changes.

REMARK 2.1. The conclusions of the above Lemmas 2.1-2.4 remain in force when dealing with a single set

$$
1>\alpha_{1}>\alpha_{2}>\cdots>\alpha_{r}>0 \quad(r=2 m-1 \text { or } 2 m) .
$$

For $r=2 m-1$, we form the sets

$$
S_{1}=\left\{1, \alpha_{2}, \alpha_{4}, \cdots, \alpha_{2 m-2}, 0\right\}, S_{2}=\left\{\alpha_{1}, \alpha_{3}, \cdots, \alpha_{2 m-1}\right\} .
$$

Then construct $F(x)$ with unit jumps at 0 and 1 and of size 2 at the points $\alpha_{2 i} ; G$ is determined with jumps of size 2 at $\alpha_{2 i-1}$. Again $F(x)-G(x)$ changes sign at most $2 m-1$ times. An analogous result applies for the case $r=2 m$.

The following lemma complements the information encompassed in Lemmas 2.1-2.4.

LEMMA 2.5. Let $H(x) \equiv 0$ be right continuous on $[0,1]$ with $H(-z)=$ $H(1+z)=0$ for $z>0$, and suppose

$$
\int_{-\epsilon}^{1+\epsilon} u_{i}(x) d H(x)=0, \quad i=1,2, \cdots, n,
$$

where $v_{i}(x)=d u_{i}(x) / d x, i=1,2, \cdots, n$, constitute a Tchebycheff system on $[0,1]$. Then $H$ changes sign at least $n$ times.

PROOF. Integrating by parts in (2.4), the boundary terms vanish yielding

$$
\int_{0}^{1} H(x) v_{i}(x) d x=0, \quad i=1,2, \cdots, n .
$$

Suppose $H$ changes sign only $r \leqslant n-1$ times on $[0,1]$. Let the sign changes occur at the points $\zeta_{1}, \zeta_{2}, \cdots, \zeta_{r}$. It is possible to construct a real continuous polynomial $v(x)=\sum_{i=1}^{n} a_{i} v_{i}(x)$ (see [7, Chapter 1]) which changes sign exactly at the $\zeta_{i}$ and nowhere else. The relations (2.5) entail $\int_{0}^{1} v(x) H(x) d x=0$.

On the other hand, the integrand is one sign and nonzero on sets of posi- 
tive measure. The obvious contradiction implies the desired conclusion of the lemma.

The result of the lemma does not necessarily apply if $\left\{v_{i}(x)\right\}$ constitute a "weak" Tchebycheff system.

3. Interpolation by generalized perfect splines. We have prepared the background ingredients for the proof of the principal interpolation Theorem 4. There are two main steps in the analysis. We first establish an essential global one to oneness of a certain nonlinear mapping in Euclidean $m$ space, $E^{m}(m=n+$ $r+1)$. The second phase involves a topological argument ultimately demonstrating the fact that the mapping covers all of $E^{m}$.

For later purposes it is convenient to develop the results in a more general framework. Let $\left\{u_{i}(x)\right\}_{1}^{n+r+1}$ comprise a family of continuous differentiable functions defined on $[0,1]$ and set $v_{i}(x)=d\left[u_{i}(x)\right] / d x, i=1,2, \cdots, n+$ $r+1$. Let $A=\left\|A_{i k}\right\|$ be an $(n+r+1) \times n$ matrix $(i=1,2, \cdots, n+r+1$; $k=1,2, \cdots, n)$ with the property that all determinants of the form

$$
\left|\begin{array}{cccccc}
A_{1,1} & A_{1,2}, & \cdots, & A_{1, n} & v_{1}\left(\eta_{1}\right), \cdots, v_{1}\left(\eta_{s}\right) \\
A_{2,1} & A_{2,2}, & \cdots, & A_{2, n} & v_{2}\left(\eta_{1}\right), \cdots, v_{2}\left(\eta_{s}\right) \\
\cdot & \cdot & & \cdot & \cdot & \cdot \\
\cdot & \cdot & & \cdot & \cdot & \cdot \\
\cdot & \cdot & & \cdot & \cdot & \cdot \\
A_{n+s, 1} & A_{n+s, 2}, & \cdots, & A_{n+s, n} & v_{n+s}\left(\eta_{1}\right), \cdots, v_{n+s}\left(\eta_{s}\right)
\end{array}\right|
$$

are of one strict sign for arbitrary choices $0<\eta_{1}<\eta_{2}<\cdots<\eta_{s}<1$ and $0 \leqslant s \leqslant r+1$. ( $s=0$ means that no $\eta_{i}$ are involved.) These requirements are henceforth in force unless stated otherwise.

That these sorts of conditions on $A$ and $\left\{v_{i}(x)\right\}$ are fulfilled in many natural cases will be adequately demonstrated later.

Consider now a mapping $\Phi$ of $\Delta_{n+r+1} \subset E^{n+r+1}$ into $E^{n+r+1}$ whose domain $\Delta_{n+r+1}$ consists of the points of the form $\{\mathbf{a}, c, \xi\}=\left\{a_{1}, a_{2}, \cdots, a_{n}, c\right.$, $\left.\xi_{1}, \xi_{2}, \cdots, \xi_{r}\right\}$ where all the coordinates are real and $\xi_{i}$ satisfy $0 \leqslant \xi_{1} \leqslant \xi_{2} \leqslant$ $\cdots \leqslant \xi_{r} \leqslant 1$. The simplex of $\xi$ 's alone is denoted by $\Sigma_{r}$. The explicit prescription of $\Phi=\Phi(\mathrm{a}, c, \xi)=\left(\Phi_{1}, \Phi_{2}, \cdots, \Phi_{n+r+1}\right)$ is

$$
\begin{array}{r}
\Phi_{i}=\sum_{j=1}^{n} A_{i j} a_{j}+c\left[u_{i}(1)+(-1)^{r+1} 2 \sum_{j=1}^{r}(-1)^{j} u_{i}\left(\xi_{j}\right)+(-1)^{r+1} u_{i}(0)\right], \\
i=1,2, \cdots, n+r+1 .
\end{array}
$$


(When some $\xi_{i}$ 's are coalesced or some $\xi_{i}$ 's coincide with 0 or 1 , then the second sum actually involves less than $r$ summands.)

THEOREM 3.1. The mapping $\Phi(\mathrm{a}, c, \xi)$ defined on $\Delta_{n+r+1}$ is $1: 1$ to the extent that the vector identity

$$
\Phi(\mathrm{a}, c, \xi)=\Phi(\tilde{\mathrm{a}}, \tilde{c}, \tilde{\xi}) \text { subject to } c^{2}+\tilde{c}^{2}>0 \text { and }
$$

where $\xi$ involves $s(s \leqslant r)$ distinct components and $\tilde{\xi}$

at most $s$ distinct components entails $\mathbf{a}=\tilde{\mathbf{a}}, c=\tilde{c}$, $\xi=\widetilde{\xi}$.

When $c=\tilde{c}=0$ and $\Phi(\mathbf{a}, 0, \xi)=\Phi(\widetilde{a}, 0, \tilde{\xi})$ holds then $\mathbf{a}=\tilde{\mathbf{a}}$ and $\Phi(\mathrm{a}, 0, \xi)$ is manifestly independent of $\xi$.

Proof. Suppose (3.3) holds and for simplicity assume that $\xi$ and $\tilde{\xi}$ both involve $r$ distinct components. (for the case where $\boldsymbol{\xi}$ and/or $\tilde{\xi}$ have coincident components, the analysis is easily adapted, mutatis mutandis.)

Case 1: $\tilde{c} \tilde{c}>0$. In this situation without restricting generality we may divide (3.2) by $\tilde{c}$ and assume $c>1=\tilde{c}$. Then (3.3) reduces to

$$
\Phi_{i}(\mathrm{a}, c, \xi)-\Phi_{i}(\tilde{\mathrm{a}}, 1, \tilde{\xi})=\sum_{k=1}^{n} A_{i k}\left(a_{k}-\tilde{a}_{k}\right)
$$

$$
\begin{aligned}
& +c\left[u_{i}(1)+(-1)^{r+1} 2 \sum_{j=1}^{r}(-1)^{j} u_{i}\left(\xi_{j}\right)+(-1)^{r+1} u_{i}(0)\right] \\
& -\left[u_{i}(1)+(-1)^{r+1} 2 \sum_{j=1}^{r}(-1)^{j} u_{i}\left(\tilde{\xi}_{j}\right)+(-1)^{r+1} u_{i}(0)\right] .
\end{aligned}
$$

Identifying the points $\left\{\xi_{i}\right\}$ and $\left\{\tilde{\xi}_{i}\right\}$ properly with $\left\{\alpha_{i}\right\}$ and $\left\{\beta_{i}\right\}$ in Lemmas 2.1 or 2.3 , we may write (3.4) in the form

$$
\sum_{k=1}^{n} A_{i k}\left(a_{k}-\tilde{a}_{k}\right)+\gamma \int_{-\epsilon}^{1+\epsilon} u_{i}(x)[d(F(x)-G(x))]=0
$$

where $\gamma$ is an appropriate nonzero constant. Integration by parts in (3.5), $i=$ $1,2, \cdots, n+r+1$, produces

$$
\begin{aligned}
\sum_{k=1}^{n} A_{i k}\left(a_{k}-\tilde{a}_{k}\right)+\gamma \int_{0}^{1} v_{i}(x)[F(x)-G(x)] d x & =0, \\
i & =1,2, \cdots, n+r+1 .
\end{aligned}
$$

On the basis of Lemmas 2.1 or 2.3 we know that either $H(x)=F(x)-G(x)$ changes sign $p$ times with $p \leqslant r$ or $H(x) \equiv 0$. Consider the first possibility. Let $s_{1}<s_{2}<\cdots<s_{p}$ be places where $H(x)$ changes sign. Now, determine $\left\{\lambda_{i}\right\}$ not all zero, to satisfy 


$$
\sum_{i=1}^{n+p+1} \lambda_{i} A_{i k}=0, \quad k=1,2, \cdots, n,
$$

$$
\sum_{i=1}^{n+p+1} \lambda_{i} v_{i}\left(s_{\mu}\right)=0, \quad \mu=1,2, \cdots, p(p \leqslant r) .
$$

Explicitly $\lambda_{i}$ (apart from a constant multiplier) are the minors with alternating signs obtained by eliminating successive columns from the matrix

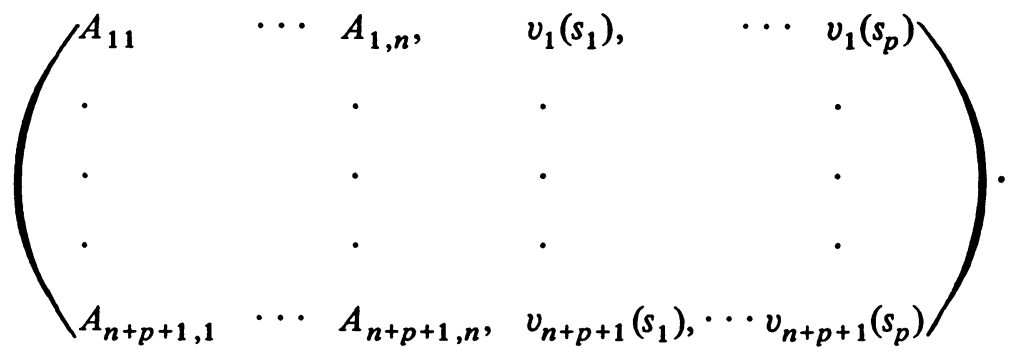

Obviously, by condition (3.1), $\lambda_{n+p+1} \neq 0$. We claim that $v(x)=\sum_{i=1}^{n+p+1} \lambda_{i} v_{i}(x)$ necessarily changes sign at $s_{1}, s_{2}, \cdots, s_{p}$ and keeps a constant sign for $s_{\mu}<$ $x<s_{\mu+1}$. The latter conclusion is immediate since $v(x)$ vanishing at such an $x$ is incompatible with the requirement of (3.1). With the stipulations of (3.7) and (3.8), it can be established (paralleling the analysis in Karlin and Studden [1966, Chapter 1] that if nodal zeros of $\sum_{i=1}^{n+p+1} \lambda_{i} v_{i}(x)$ are counted once and nonnodal zeros twice then the maximum number of zeros in this count for $v(x)=$ $\Sigma \lambda_{i} v_{i}(x)$ is at most $p$. With this property we see that $v(x)$ actually changes sign at each $s_{\mu}$.

A more direct proof that $v(x)$ changes sign runs as follows. Suppose to the contrary that $v\left(s_{i_{0}}\right)=0$ but $v(x)$ has one sign (say $>0$ ) in a neighborhood of $s_{i_{0}}$. Then determine $\mu_{i}$ satisfying

$$
\epsilon \sum_{i=1}^{n+p} \mu_{i} A_{i k}=0 \quad \text { and } \epsilon \sum_{i=1}^{n+p} \mu_{i} v_{i}\left(s_{\mu}\right)=0, \quad \mu \neq i_{0}
$$

(notice we have involved $n+p$ variables $\mu_{i}$ rather than $n+p+1$ occurring in (3.7) and (3.8)) and determine the sign of $\epsilon$ such that $\epsilon \sum_{i=1}^{n+p} \mu_{i} v_{i}\left(s_{\mu_{0}}\right)<0$. Obviously (setting $\mu_{n+p+1}=0$ ), we have

$$
\sum_{i=1}^{n+p+1}\left(\lambda_{i}+\epsilon \mu_{i}\right) A_{i k}=0, \quad k=1, \cdots, n,
$$

and $\sum_{i=1}^{n+p+1}\left(\lambda_{i}+\epsilon \mu_{i}\right) v_{i}(x)$ vanishes at $p+1$ distinct points for $\epsilon$ small enough. This is impossible because of condition (3.1).

The obvious combination of (3.7) and (3.8) leads to 


$$
0=\sum_{i=1}^{n+p+1} \lambda_{i} \sum_{k=1}^{n} A_{i k}\left(a_{k}-\tilde{a}_{k}\right)+\gamma \int_{0}^{1} v(x) H(x) d x=\gamma \int_{0}^{1} v(x) H(x) d x
$$

However, $v(x) H(x)$ is not identically zero and keeps a constant sign throughout $[0,1]$ and accordingly (3.9) is manifestly absurd. We may therefore conclude that $H(x) \equiv 0$, i.e., $\xi_{i}=\widetilde{\xi}_{i}$ and $c=\widetilde{c}=1$. The remaining equations $\sum_{k=1}^{n} A_{i k}\left(a_{k}-\tilde{a}_{k}\right)=0, i=1,2, \cdots, n$, admit only the trivial solution in view of assumption (3.1).

Case 2: $c \tilde{c}<0$. The analysis paraphrases the preceding invoking Lemmas 2.2 and Lemma 2.4 in place of Lemmas 2.1 and 2.3.

Case 3: $c \tilde{c}=0, c^{2}+c^{2}>0$. Remark 2.1 of $\S 2$ is relevant here.

Case 4: $c=\tilde{c}=0$. The proof of Theorem 3.1 is complete except for the last statement. But that fact is immediate since $A$ certainly has rank $n$ and therefore $A(\mathbf{a}-\widetilde{a})=0$ entails $\mathbf{a}=\widetilde{a}$.

Consider now the restriction $F$ of the mapping $\phi$ by holding $c$ fixed at $c=1$. More specifically, we define $\phi_{+}=\left(\phi_{1}^{+}, \cdots, \phi_{n+r+1}^{+}\right)$on $\Delta \subset E^{n+r}$ where

$$
\Delta=\{\mathrm{a}, \xi\}=\left\{a_{1}, a_{2}, \cdots, a_{n}, \xi_{1}, \xi_{2}, \cdots, \xi_{r}\right\}
$$

and $\left\{\xi_{i}\right\}$ are constrained according to $0<\xi_{1}<\xi_{2}<\cdots<\xi_{r}<1$, while $a_{i}$ are free real variables, and

$$
\begin{array}{r}
\phi_{i}^{+}(\mathrm{a}, \xi)=\sum_{k=1}^{n} A_{i k} a_{k}+\left[u_{i}(1)+2(-1)^{r+1} \sum_{j=1}^{r}(-1)^{j} u_{i}\left(\xi_{j}\right)+(-1)^{r+1} u_{i}(0)\right] \\
i=1,2, \cdots, n+r+1 .
\end{array}
$$

Next, transfer the mapping so that the image is on the surface $S^{n+r+1}$ of the unit ball in $E^{n+r+1}$. Specifically, define

$$
f_{i}^{+}(\mathrm{a}, \xi)=\frac{\phi_{i}^{+}(\mathrm{a}, \xi)}{\sqrt{\sum_{i=1}^{n+r+1}\left[\phi_{i}^{+}(\mathrm{a}, \xi)\right]^{2}}}, \quad i=1,2, \cdots, n+r+1,
$$

and set $F^{+}=\left(f_{1}^{+}, f_{2}^{+}, \cdots, f_{n+r+1}^{+}\right)$.

Theorem 3.1 tells us that $F^{+}$is well defined continuous and a globally "1:1" mapping of $\Delta$ into $S^{n+r+1}$ under the conditions described there. The $1: 1$ assertion in a stronger form is checked as follows. Suppose $\xi \in \Sigma$ involves $s(s \leqslant r)$ distinct components and $\widetilde{\xi}$ carries at most $s$ distinct components. The identity $F^{+}(\mathbf{a}, \xi)=F^{+}(\widetilde{a}, \widetilde{\xi})$ is the same as

$$
\phi(\mathbf{a}, 1, \xi)=\gamma \phi(\widetilde{a}, 1, \widetilde{\xi})=\phi(\gamma \tilde{\mathbf{a}}, \gamma, \tilde{\xi}),
$$

where $\gamma=$ the appropriate ratio of normalizing constants. Theorem 3.1 now applies to affirm $\gamma=1, a=\tilde{a}, \xi=\tilde{\xi}$.

The closure of the simplex $\bar{\Sigma}=\left\{0 \leqslant \xi_{1} \leqslant \xi_{2} \leqslant \cdots \leqslant \xi_{r} \leqslant 1\right\}$ and the continuous extension of $F^{+}$to $\bar{\Sigma}$ done in the obvious manner causes no diffi- 
culty. It is essential also to close the domain $\Delta$ with reference to the $\left\{a_{i}\right\}$ components. We do this as follows. Consider the homeomorphism $T$ defined on $\Delta$; viz., $\left(a_{1}, a_{2}, \cdots, a_{n}, \xi_{1}, \cdots, \xi_{r}\right)$

$$
\rightarrow\left(\frac{a_{1}}{\sqrt{1+\sum_{i=1}^{n} a_{i}^{2}}}, \frac{a_{2}}{\sqrt{1+\sum_{i=1}^{n} a_{i}^{2}}}, \cdots, \frac{a_{n}}{\sqrt{1+\sum_{i=1}^{n} a_{i}^{2}}}, \xi_{1}, \cdots, \xi_{r}\right)
$$

for any $\xi \in \bar{\Sigma}$.

(Since $T$ only affects the a components we write, for brevity, $T \mathbf{a}=\mathbf{b}$ rather than $T\{a, \xi\}$.) The first $n$ components in the closure of the image of $T$ covers the corresponding unit ball, and through $T$ we will close the domain $\Delta$ in the natural way to $\bar{\Delta}$. We have mapped the space $E^{n}:\left\{\mathrm{a}=\left(a_{1}, \cdots, a_{n}\right)\right\}$ to the open sphere $T a$ and then close the image domain in the natural way by adding ideal points at infinity to $\Delta$ of dimension homeomorphic to the boundary of the unit ball in $E^{n}$ space.

The mapping $F^{+}$extends to the closure $\bar{\Delta}$ as follows. Suppose $\left\{\mathrm{a}^{(\nu)}\right.$, $\xi\} \in \Delta$ is such that $T \mathbf{a}^{(\nu)}$ tends to a boundary point say $\mathbf{b}^{*}$ (meaning that $\sum_{i=1}^{n}\left|a_{i}^{(\nu)}\right| \rightarrow \infty$ as $m \rightarrow \infty$ and $a_{i}^{(\nu)} / \sqrt{1+\sum_{i=1}^{n}\left(a_{i}^{(\nu)}\right)^{2}} \rightarrow b_{i}^{*}, i=1,2, \cdots$, $n$ ). It follows immediately from (3.12) and the special form of (3.11), that

$$
f_{i}^{+}\left(\mathrm{a}^{(\nu)}, \xi\right)=\frac{\phi_{i}^{+}\left(\mathrm{a}^{(\nu)}, \xi\right) / \sqrt{1+\sum_{i=1}^{n}\left(\mathrm{a}_{i}^{(\nu)}\right)^{2}}}{\sqrt{\sum_{i}\left[\phi_{i}^{+}\left(\mathrm{a}^{(\nu)}, \xi\right) / \sqrt{1+\sum_{i=1}^{n}\left(\mathrm{a}_{i}^{(\nu)}\right)^{2}}\right]^{2}}}
$$

converges to

$$
\phi_{i}^{+}\left(\mathrm{b}^{*}, 0, \xi\right) / \sqrt{\sum_{i=1}^{n+r+1}\left[\phi_{i}\left(\mathrm{~b}^{*}, 0, \xi\right)\right]^{2}}=\text { Definition of } F^{+}\left(\mathrm{b}^{*}, \xi\right) .
$$

The limit evaluation of $f_{i}^{+}$for $\mathbf{b}^{*}=\lim _{\nu \rightarrow \infty} T \mathrm{a}^{(\nu)}$ is manifestly independent of the approximating sequence. Note that the components in (3.13) do not depend on $\xi$. The image of this part of the boundary of $\bar{\Delta}$ is clearly homeomorphic to a simplex. Observe that $\bar{\Delta}$ now consists of the direct product of a closed unit ball of $E^{n}$ and a closed bounded simplex $\bar{\Sigma}$ in $E^{r}$.

It is elementary to check that the mapping $F^{+}$as extended in (3.13) persists as a continuous mapping defined on $\bar{\Delta}$ into $S^{n+r+1}$.

Analogous to the determination of $F^{+}$, we construct $F^{-}$corresponding to the mapping $\Phi$ with $c$ fixed at -1 . We record the preceding discussion formally as a lemma. 
Lemma 3.1. The mapping $F^{+}$(and also $F^{-}$) is defined explicitly in (3.11) where if $\left\{\mathrm{a}^{*}, \xi\right\}$ is such that $T \mathrm{a}^{*}=\mathrm{b}^{*}(T$ defined in (3.12)) lies on the surface of the unit sphere of $E^{n}$ then the determination is given by

$$
F^{+}\left(\left\{\mathrm{a}^{*}, \xi\right\}\right)=\left\{\frac{\Phi_{i}\left(\mathrm{~b}^{*}, 0, \xi\right)}{\sqrt{\sum_{i=1}^{n+r+1}\left[\phi_{i}\left(\mathrm{~b}^{*}, 0, \xi\right)\right]^{2}}}\right\}_{i=1}^{n+r+1}
$$

and this image is independent of $\xi$. The mapping $F^{+}$sends $\bar{\Delta}$ continuously into $S^{n+r+1}$ (the surface of the unit ball lying in $E^{n+r+1}$ ). Moreover, $F^{+}$is $1: 1$ except for the images of $\{a, \xi\}$ where $\xi$ lies in $\partial \bar{\Sigma}_{r}$ (the boundary of $\Sigma_{r}$ ) and for the points (3.14) which can only come from boundary points in $\bar{\Delta}$ of the form $\left\{\mathbf{b}^{*}, \xi\right\}$ provided $\mathbf{b}^{*}$ is a boundary point of $T \mathbf{a}$.

The next lemma is crucial to our deliberations.

Let $V=F^{+}(\bar{\Delta})$ (the image of $\bar{\Delta}$ under $F^{+}$in $S^{n+r+1}$ ). Regard $V$ as a topological subspace of $S^{n+r+1}$. Also let $U=F^{-}(\bar{\Delta})$.

LEMMA 3.2. $V^{\circ}=F^{+}\left(\bar{\Delta}^{\circ}\right)$ and $\partial V=F^{+}(\partial \bar{\Delta})\left(V^{\circ}\right.$ denotes the interior of $V$ and $\partial V$ the boundary of $V) . U^{\circ}=F^{-}\left(\bar{\Delta}^{\circ}\right)$ and $\partial U^{\circ}=F^{-}(\partial \bar{\Delta})$ and $V^{\circ} \cap$ $U^{\circ}=$ void.

Proof. The assertion follows from the invariance of domain principle (open mapping theorem) based on the fact that $F^{+}$is globally $1: 1$ on $\bar{\Delta}^{\circ}$ as affirmed in Theorem 3.1. Note also that the boundary maps exclusively into the boundary of the image set. It is then evident that this principle identifies $V^{\circ}=F^{+}\left(\bar{\Delta}^{\circ}\right)$ and $\partial V=F^{+}(\partial \bar{\Delta})$. The fact that $V^{\circ} \cap U^{\circ}$ is void is also a direct consequence of the global 1:1 conclusion of Theorem 3.1.

The next lemma provides an important identification of $\partial V$ and $\partial U$.

LEMMA 3.3. $\partial V=\partial U$.

Proof. Note that the boundary of $\bar{\Delta}$ consists of points $\{a, \xi\}$ where either $T \mathrm{a}=\mathrm{b}$ lies on the boundary of $B^{n}$ (the unit ball in $E^{n}$ ) and/or where at least $\xi_{i}=\xi_{i+1}$ for some $i$, or $\xi_{j}=0$ or $\xi_{j}=1$ for some $j$.

We consider all these possibilities in order.

Case 1. If $T \mathrm{a}=\mathrm{b} \in \partial\left(B^{n}\right)$, then consulting (3.14) reveals that $F^{+}(\{\mathrm{a}, \xi\})=$ $F^{-}(\{a, \xi\})$.

Case 2. $\{a, \xi\}$ has $a_{k}$ all finite but $\xi_{1}=0$ such that $0=\xi_{1}<\xi_{2}<$ $\xi_{3}<\cdots<\xi_{r}<1$. Now specify $\eta_{r}=1, \eta_{r-1}=\xi_{r}, \cdots, \eta_{1}=\xi_{2}$. 
Direct verification yields

$$
F^{+}(\{\mathbf{a}, \xi\})=F^{-}(\{\mathbf{a}, \eta\}) .
$$

Case 3. $\{\mathrm{a}, \xi\}$ has all $a_{k}$ finite but $0<\xi_{1}<\xi_{2}<\cdots<\xi_{r}=1$. Now specify $0=\eta_{1}, \eta_{i+1}=\xi_{i}, i=1,2, \cdots, r-1$, and again (3.15) ensues.

Case 4. Suppose $\{\mathrm{a}, \xi\}$ is such that all $a_{k}$ are finite but for some $i, \xi_{i}=$ $\xi_{i+1}$. Now choose $\eta$ to have $\eta_{r}=1, \eta_{r-1}=\xi_{r}, \cdots, \eta_{i+1}=\xi_{i+2}, \eta_{i}=\xi_{i-1}$, $\cdots, \eta_{2}=\xi_{1}, \eta_{1}=0$. Direct evaluation yields

$$
F^{+}(\{\mathbf{a}, \xi\})=F^{-}(\{\mathbf{a}, \eta\}) .
$$

Cases 2-4 cover all the $r-1$ dimensional faces of the boundary for the simplex $0 \leqslant \xi_{1} \leqslant \xi_{2} \leqslant \cdots \leqslant \xi_{r} \leqslant 1$ which establishes the validity of $\partial V=\partial U$ in these cases. The property extends to all of $V$ by continuity. Thus $\partial V \subset \partial U$ and from symmetry considerations we deduce $\partial V=\partial U$. The lemma is proved.

COROLLARY 3.1. $V=F^{+}(\bar{\Delta})$ and $U=F^{-}(\bar{\Delta})$ have the same boundary and their interiors are disjoint.

Our next objective is the proof of

THEOREM 3.2. The mapping $F=F^{+} \cup F^{-}$is onto all of $S^{n+r+1}$.

In order to validate this theorem we make the important induction assumption that the corresponding result holds in the presence of at most $r-1$ knots. Thus, we impose

INDUCTION Assumption. The image set $\Gamma$ on $S^{n+r+1}$ for mapping of $F$ : $F^{+} \cup F^{-}$of all points $\{\mathrm{a}, \xi\} \in \bar{\Delta}$ involving at most $r-1$ knots (i.e., at most $r-1$ distinct $\xi_{i}$ are allowed) is homeomorphic to the surface of a sphere of dimension $n+r$.

A little reflection reveals (because of Lemma 3.3) that

$$
\Gamma=\partial V=F^{+}(\partial \Delta)
$$

so that the induction assumption asserts that $F^{+}(\partial \bar{\Delta})$ is homeomorphic to a sphere of dimension $n+r$.

The induction assumption is patently valid with no knots, as the image $F^{+}\left(T_{a}\right)$ is obviously homeomorphic to a solid sphere of dimension $n$.

The next lemma is vital for the proof of Theorem 3.2.

LEMMA 3.4. The image of $F^{+}(\bar{\Delta})$ is homeomorphic on $S^{n+r+1}$ to a simplex of dimension $n+r+1$. The same is true for $F^{-}(\bar{\Delta})$. 
Proof. Take a point interior to $F^{+}(\bar{\Delta})=V$ and map by a stereographic projection the set $V$ onto $K \subset E^{n+r+1}$. Consider the complement of $K$ in $E^{n+r+1}$. It cannot have two components because then $\partial K$ is not homeomorphic to a sphere of dimension $n+r$ contradicting the induction assumption (3.17). We also claim that $K$ is simply connected (i.e. any closed surface in $K^{c}$ can be contracted continuously to a point persisting inside $K^{c}$ ). If not, we would again contradict the induction assumption stipulating that $\partial K$ is homeomorphic to a sphere of dimension $n+r$. Thus Lemma 3.4 is established.

Proof OF TheOREM 3.2. Let $Z$ be the complement of $V=F^{+}(\bar{\Delta})$ in $S^{n+r+1}$. By virtue of Lemma 3.5 we know that $Z$ is open and simply connected. Of course $F\left(\bar{\Delta}^{\circ}\right)=U^{\circ} \subset Z$ as attested to in Corollary 3.1. Suppose $U^{\circ}$ is properly contained in $Z$. Take $p \in Z$ but not in $U^{\circ}$. Since $Z$ is connected we can obviously join $p$ to a point of $U^{\circ}$ by a curve contained strictly inside $Z$ and therefore not touching $V$. But, the curve crosses $\partial U$ and since $\partial U=$ $\partial V$ (Lemma 3.3) an absurdity results. Thus we have established $U^{\circ}=Z$ and the proof of the theorem is complete.

With Theorem 3.2 established, the induction step is advanced to the case of $r$ knots.

We essentially have now the main theorem.

THEOREM 3.3. The mapping $\Phi$ defined on $\Delta=\Delta_{n+r+1}$ covers $E^{n+r+1}$.

Proof. Any point $\mathbf{x} \neq 0$ of $E^{n+r+1}$ is manifestly a constant multiple of a point $p$ of $S^{n+r+1}$. In the case where $p$ belongs to $F^{+}\left(\bar{\Delta}^{\circ}\right)$ or $F\left(\bar{\Delta}^{\circ}\right)$ we then obtain that $\Phi(\{\mathbf{a}, c, \xi\})=\mathbf{x}$ for some unique $c \neq 0$ and $\xi$ possesses $r$ distinct components.

According to the characterization of the boundary $F^{+}(\partial \bar{\Delta})$ depicted in the proof of Lemma 3.3, when $p \in F^{+}(\partial \bar{\Delta})$ we can achieve its image by either the choice of $c=0$ and appropriate determination of $\left\{a_{i}\right\}$ or where $\xi$ involves fewer than $r$ knots and $c \neq 0$.

Examination of the proof also reveals the uniqueness assertion as stated in the Theorem. It remains only to prove the last statement of Theorem 4. Suppose the data $\left\{\alpha_{i}\right\}$ consists of $n+r$ zeros and $c \neq 0$ and assume to the contrary that a solution exists with $\leqslant r-1$ knots. The argument (a much simpler variant) of Theorem 3.1 of (3.5) produces a contradiction so any solution has $r$ distinct knots and Theorem 3.1 applies to guarantee uniqueness.

4. Interpolation by perfect splines. Let $0 \leqslant x_{1}<x_{2}<\cdots<x_{n+r+1} \leqslant 1$ be prescribed with corresponding real data $\left\{\alpha_{i}\right\}_{1}^{n+r+1}$, not all zero. This section 
is devoted to the proof of Theorem 1 stated in $\S 1$. The objective is to establish the existence of a perfect spline of the form

$$
P(x)=\sum_{j=0}^{n-1} a_{j} x^{j}+c\left[x^{n}+2 \sum_{i=1}^{s}(-1)^{i}\left(x-\xi_{i}\right)_{+}^{n}\right]
$$

with $s \leqslant r$ satisfying

$$
P\left(x_{k}\right)=\alpha_{k}, \quad k=1,2, \cdots, n+r+1 .
$$

More specifically, we seek to determine real constants $\left\{a_{i}\right\}_{0}^{n-1}, c$ and $0<\xi_{1}<$ $\xi_{2}<\cdots<\xi_{s}<1 \quad(s \leqslant r)$ such that (4.2) holds. Reference to Theorem 4 suggests identifying $A_{k, j+1}=x_{k}^{j}$ and $\tilde{u}_{k}(\eta)=\left(\eta-1+x_{k}\right)_{+}^{n}$ such that the conditions (4.2) become (with $\eta_{\mu}=1-\xi_{\mu}$ )

$$
\sum_{j=1}^{n} A_{k j} a_{j}+c\left[\tilde{u}_{k}(1)+2 \sum_{\mu=1}^{s}(-1)^{j} \tilde{u}_{k}\left(\eta_{\mu}\right)\right]=\alpha_{k}, \quad k=1,2, \cdots, n+r+1 .
$$

(Note that $\tilde{u}_{k}(0)=0$ so this term has been omitted without repercussions.)

Direct appeal to Theorem 3.3 (cf. Theorem 4 of $\S 1$ ) is not permissible since the requirements of (3.1) are not fulfilled. Actually the determinant (3.1) in the case at hand keeps one sign, but not necessarily strictly. To overcome this difficulty we invoke a standard smoothening procedure. The function $\tilde{u}_{k}(\xi)$ may be replaced by

$$
u_{k}(\xi ; \epsilon)=\frac{1}{\sqrt{2 \pi \epsilon}} \int_{-\infty}^{\infty} \exp \left[-\frac{(\xi-\zeta)^{2}}{2 \epsilon}\right]\left(\zeta-1+x_{k}\right)_{+}^{n} d \zeta
$$

defined on $-\infty<\xi<\infty$.

From integration by parts note that

$$
v_{k}(\xi ; \epsilon)=\frac{d u_{k}}{d \xi}(\xi ; \epsilon)=\frac{n}{\sqrt{2 \pi \epsilon}} \int_{-\infty}^{\infty} \exp \left[-\frac{(\xi-\zeta)^{2}}{2 \epsilon}\right]\left(\zeta-1+x_{k}\right)_{+}^{n-1} d \zeta
$$

The kernel $\Phi_{n-1}(x, \eta)=(x-\eta)_{+}^{n-1}$ is totally positive while the Gaussian kernel $G(\xi, \zeta)=\exp \left[-(\xi-\zeta)^{2} / 2 \epsilon\right]$ is extended totally positive on $-\infty<\xi$, $\zeta<\infty$, (consult [3, e.g. p. 512], on these matters).

We check the requirements of (3.1).

LEMMA 4.1. Let $s \leqslant r+1$ and let $\left\{\gamma_{i}\right\}_{i=1}^{s}$ be $s$ ordered points in $(0,1)$, $0<\gamma_{1}<\gamma_{2}<\cdots<\gamma_{s}<1$. Then the $(n+s) \times(n+s)$ determinant 
(4.5)

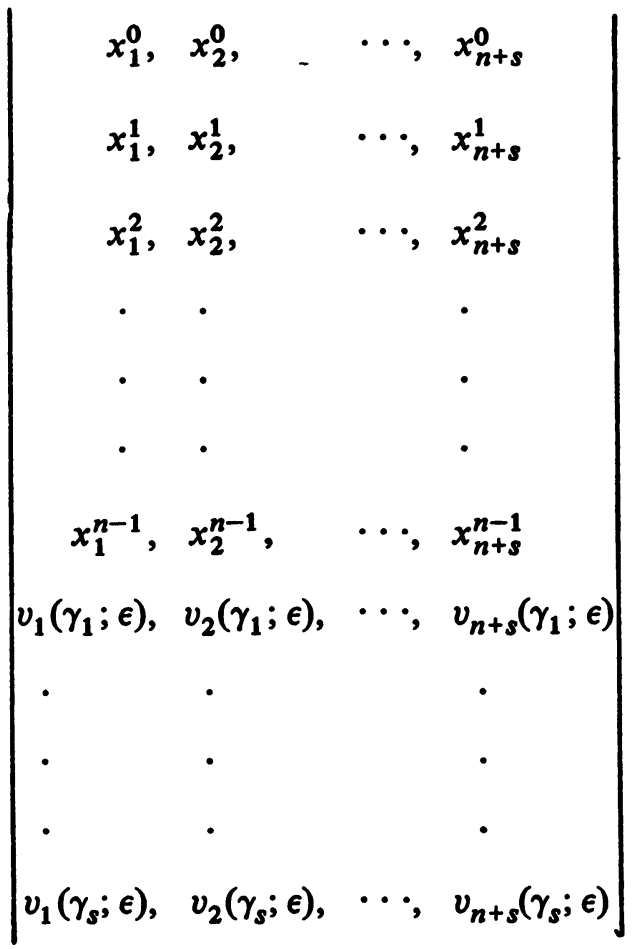

is nonvanishing for each $\epsilon>0$.

ProOF. The preceding determinant equals

(4.6)

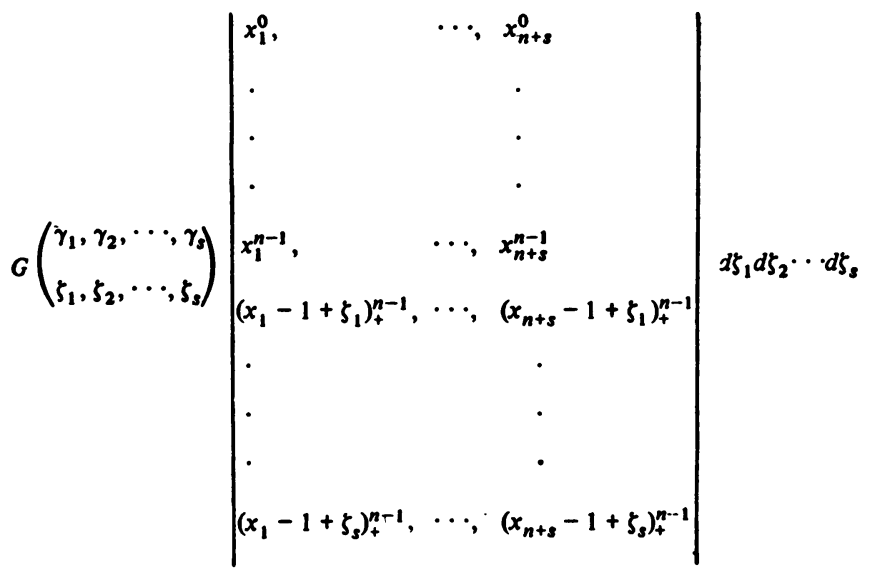

where

$$
G\left(\begin{array}{l}
\gamma_{1}, \cdots, \gamma_{s} \\
\zeta_{1}, \cdots, \zeta_{s}
\end{array}\right)=\operatorname{det}\left[G\left(\gamma_{i}, \zeta_{j}\right)\right]
$$


for

$$
G(\gamma, \zeta)=(n / \sqrt{2 \pi \epsilon}) \exp \left(-(\gamma-\zeta)^{2} / 2 \epsilon\right)
$$

The formula can be derived via the Laplace determinant expansion with further reliance on the Cauchy-Binet identity. Or it is a special circumstance of the composition formula [3, p. 17].

Owing to the specifications $0 \leqslant x_{1}<x_{2}<\cdots<x_{n+r+1} \leqslant 1$, the integration domain can be reduced to $0 \leqslant \zeta_{1}<\cdots<\zeta_{s} \leqslant 1$ since in all other circumstances the final determinant appearing in the integrand (4.6) is zero. Indeed, where $\zeta_{1}<0$, the $(n+1)$ th row displays all zero entries and where $\zeta_{s}>1$ the bottom row can be expressed as a linear combination of the first $n$ rows and again the determinant vanishes. Thus (4.6) becomes

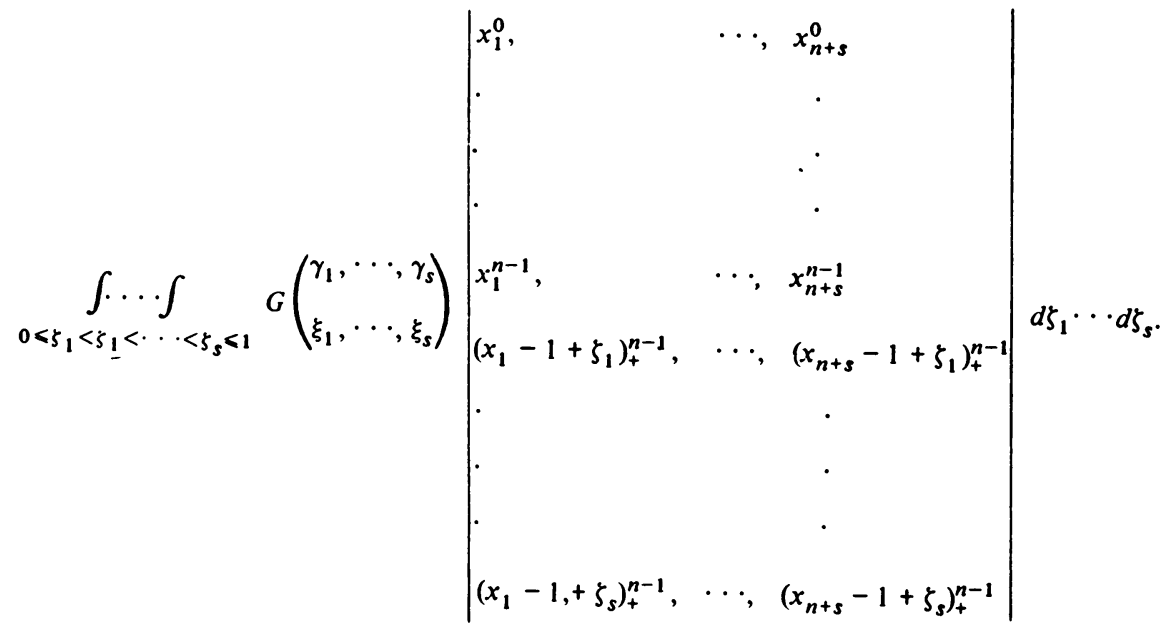

The second determinant in the integral is of the form of those discussed in $\$ 2$ of Chapter 10 in [3]. Theorems 2.1 and 2.2 therein show that over the region of integration, this determinant is always of one sign and strictly nonvanishing on sets of positive measure. The kernel $G$ is extended totally positive, which means that

$$
G\left(\begin{array}{l}
\gamma_{1}, \cdots, \gamma_{s} \\
\zeta_{1}, \cdots, \zeta_{s}
\end{array}\right)
$$

is always positive. No cancellation is therefore possible in (4.6) and we conclude that this integral is nonzero of a predictable sign. The lemma is proved.

The content of Lemma 4.1 permits implementation of Theorem 3.3 which produces the result.

LEMMA 4.2. There exists $a$ set of real constants $\left\{a_{i}(\epsilon)\right\}, c(\epsilon)$ and $\left\{\zeta_{j}(\epsilon)\right\}$, 
$0<\zeta_{1}(\epsilon)<\zeta_{2}(\epsilon)<\cdots<\zeta_{s}(\epsilon)<1(s \leqslant r)$, depending on $\epsilon>0$, satisfying

$$
\sum_{i=0}^{n-1} a_{i}(\epsilon) x_{k}^{i}+c(\epsilon)\left[u_{k}(1 ; \epsilon)+2 \sum_{j=1}^{s}(-1)^{s+j+1} u_{k}\left(\zeta_{j}(\epsilon) ; \epsilon\right)\right.
$$

$$
\begin{aligned}
& \left.+(-1)^{s+1} u_{k}(0 ; \epsilon)\right]=\alpha_{k}, \\
& \quad k=1,2, \cdots, n+r+1 .
\end{aligned}
$$

It remains to send $\epsilon \downarrow 0$ and achieve (4.2). The next two lemmas justify this process. For this purpose note first the following facts.

(i) It is a familiar smoothing property of the Gaussian kernel that $u_{k}(\xi, \epsilon) \rightarrow$ $\left(\xi-1+x_{k}\right)_{+}^{n}, n \geqslant 1$, uniformly for $0 \leqslant \xi \leqslant 1$ as $\epsilon \downarrow 0$.

(ii) By passing to a subsequence if need be, we may assume that $\zeta_{j}(\epsilon)$ (occurring in (4.7)) converge to $\xi_{j}$ and $0 \leqslant \xi_{1} \leqslant \xi_{2} \leqslant \cdots \leqslant \xi_{s} \leqslant 1$. Corresponding blocks of coincident groups of $\xi$ values and equalities to 0 or 1 causes the sum $x_{k}^{n}+(-1)^{s+1} 2 \Sigma_{j=1}^{s}(-1)^{j}\left(\xi_{j}-1+x_{k}\right)_{+}^{n}$ to reduce and of course $u_{k}(0, \epsilon) \rightarrow$ 0 as $\epsilon \downarrow 0$.

LEMMA 4.3. The constants $\left\{a_{i}(\epsilon)\right\}$ and $c(\epsilon)$ can be chosen to converge to finite limits as $\epsilon \downarrow 0$.

Proof. Several cases are considered.

Case 1. If all $\left\{a_{i}(\epsilon)\right\}_{0}^{n-1}, c(\epsilon)$ are bounded as $\epsilon \downarrow 0$ we may suppose that they (or their subsequences) converge to limits $\left\{a_{i}\right\}, c$ as $\epsilon \downarrow 0$ and (4.2) is established.

Case 2. Suppose $c(\epsilon)$ is unbounded and $\max _{0 \leqslant i \leqslant n-1}\left|a_{i}(\epsilon)\right| / c(\epsilon) \rightarrow 0$ for some subsequence of $\epsilon \downarrow 0$. Then dividing (4.7) by $c(\epsilon)$ and letting $\epsilon \downarrow 0$ gives (with the notation $\eta_{i}=1-\xi_{i}$ ),

$$
x_{k}^{n}-2\left(x_{k}-\eta_{1}\right)_{+}^{n}+2\left(x_{k}-\eta_{2}\right)_{+}^{n}-\cdots+2(-1)^{\mu}\left(x_{k}-\eta_{\mu}\right)_{+}^{n}=0
$$

where $\mu \leqslant r, k=1,2, \cdots, n+r+1$ and $1>\eta_{\mu}>\cdots>\eta_{2}>\eta_{1}>0$.

The equations assert that $x^{n}+2 \Sigma_{i=1}^{\mu}(-1)^{i}\left(x-\eta_{i}\right)_{+}^{n}$ vanishes at $x=x_{1}$, $x_{2}, \cdots, x_{n+r+1} \cdot n-1$ differentiations and Rolle's theorem implies that $x+$ $2 \Sigma_{i=1}^{\mu}(-1)^{i}\left(x-\eta_{i}\right)_{+}^{1}$ vanishes at least $r+2 \geqslant \mu+2$ times in $(0,1)$ which is impossible.

Case 3. For at least one integer $d, a_{d}(\epsilon)$ is unbounded along a subsequence of $\epsilon$ tending to zero and $c(\epsilon) / a_{d}(\epsilon) \rightarrow 0$ as $\epsilon \downarrow 0$ along a subsequence.

In this case dividing through by $\max _{1 \leqslant i \leqslant n-1}\left|a_{i}(\epsilon)\right|$ in (4.7) and letting $\epsilon \downarrow 0$ appropriately, we get 


$$
\sum_{i=0}^{n-1} b_{i} x_{k}^{i}=0, \quad k=1,2, \cdots, n+r+1
$$

We produced in (4.9) a nontrivial polynomial of degree $\leqslant n-1$ vanishing for at least $n+1$ points, an obvious absurdity.

Case 4. For some index $i, a_{i}(\epsilon)$ is unbounded but $c(\epsilon) / \max _{0 \leqslant i \leqslant n-1}\left|a_{i}(\epsilon)\right|$ is bounded away from zero and infinity.

By passing to a subsequence we extract a spline

$$
\sum_{i=0}^{n-1} b_{i} x^{i}+x^{n}+2 \sum_{j=1}^{\mu}(-1)^{j}\left(x-\eta_{j}\right)_{+}^{n}
$$

(with $b_{i}$ real) vanishing for $x=x_{1}, x_{2}, \cdots, x_{n+r+1}$ and where $r \geqslant \mu$.

Differentiating (4.10) successively and invoking repeatedly Rolle's theorem we deduce that

$$
x+e+\sum_{j=1}^{\mu} 2(-1)^{j}\left(x-\eta_{j}\right)_{+}^{1} \quad(e \text { is real })
$$

vanishes at least $r+2$ times in $[0,1]$. But $r+2 \geqslant \mu+2$ and this is manifestly impossible.

The proof of Lemma 4.3 is complete.

Summarizing the discussion of Lemma 4.3 we have demonstrated the existence fact of Theorem 1 for the case of distinct interpolating points. The extension of the existence for coincident blocks of $x$ 's obeying the stipulations set forth in Theorem 1 is carried out by a routine approximation argument repeating suitably the analysis of the cases in Lemma 4.3. The proof of Theorem 1 is complete.

The perturbations can also be done in the following manner which is more convenient for other purposes. Define

$$
A_{k, j+1}(\epsilon)=\frac{1}{\sqrt{2 \pi \epsilon}} \int_{-\infty}^{\infty} \exp \left[-\zeta^{2} / 2 \epsilon\right]\left(x_{k}-\zeta\right)^{j} d \zeta, \quad j=0,1, \cdots, n-1,
$$

$$
=\frac{1}{\sqrt{2 \pi \epsilon}} \int_{-\infty}^{\infty} \exp \left[-\left(x_{k}-\eta\right)^{2} / 2 \epsilon\right] \eta^{j} d \eta
$$

and as in (4.3) we write

$$
u_{k}(\xi, \epsilon)=\frac{1}{\sqrt{2 \pi \epsilon}} \int_{-\infty}^{\infty} \exp \left[-\left(x_{k}-\eta\right)^{2} / 2 \epsilon\right](\xi-1+\eta)_{+}^{n} d \eta
$$

Applying the composition formula [3, p. 76], we obtain the identity 


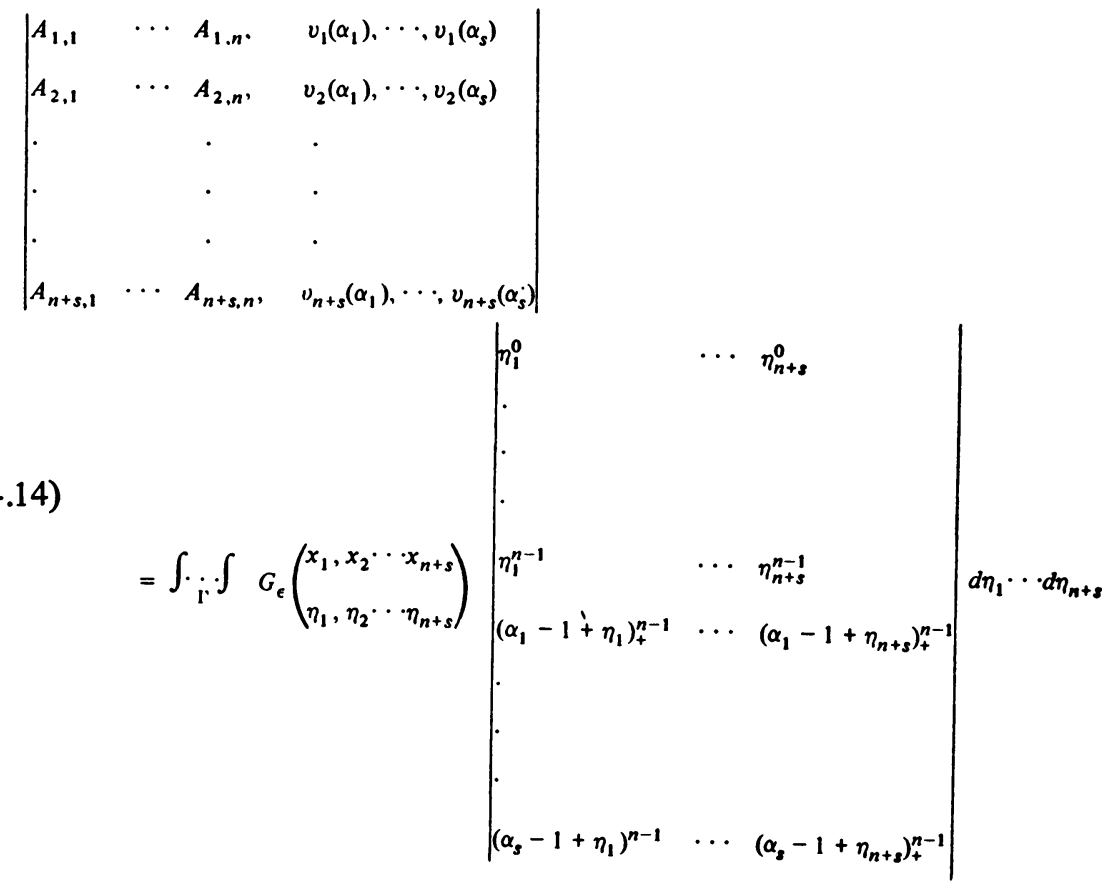

for any

$$
0<\alpha_{1}<\alpha_{2}<\cdots<\alpha_{s}<1 \text {, }
$$

where the integral in (4.14) extends over the region $\Gamma: \eta_{1}<\eta_{2}<\cdots<\eta_{n+s^{*}}$ The total positivity property of the kernel $(\xi-\eta)_{+}^{n-1}$ implies since 1 $\alpha_{1}>1-\alpha_{2}>\cdots>1-\alpha_{s}>0$ that the second determinant in the integrand never changes sign for any choices of $\alpha_{i}$ satisfying (4.15) and this determinant is certainly nonzero for a set $\left(\eta_{1}, \cdots, \eta_{n+s}\right)$ of positive Lebesgue measure of the integration domain for the specifications of $\left\{\alpha_{i}\right\}$. The $G_{\epsilon}$ determinants are always strictly positive. Therefore the nondegeneracy assumption of Theorem 4 (relation (3.1)) applies to the prescriptions (4.12) and (4.13).

5. Relations of the interpolation points and knots. Let $P(x)$ be a perfect spline of degree $n$ with at most $r$ knots, constructed to interpolate the data $\left\{\alpha_{i}\right\}_{i=1}^{n+r+1}$ at the points $\left\{x_{i}\right\}_{i=1}^{n+r+1}$ (see Theorem 1).

The answer to the following questions of independent interest will play an important role in discerning the uniqueness criteria for the interpolating perfect spline:

Determine usable sufficient conditions on $\left\{\alpha_{i}\right\}$ and $\left\{x_{i}\right\}$ that assure that $P(x)$ involves precisely $r$ knots $\left\{\xi_{i}\right\}_{1}^{r}$ and where the knots are located relative 
to $x_{i}$ such that the following holds:

$$
x_{i}<\xi_{i}<x_{i+n+1}, \quad i=1,2, \cdots, r .
$$

The following terminology is also beneficial. A perfect spline $P(x)$ of degree $n$ with $r$ knots is said to oscillate maximally (abbreviated $O$. M.) if $P(x)$ vanishes simply $n+r$ times in $(0,1)$. It follows easily that if $P(x)$ oscillates maximally, then $P^{\prime}(x), P^{\prime \prime}(x), \cdots$ do likewise 0 . M.

We will find that if the data $\left\{\alpha_{i}\right\}_{1}^{n+r+1}$ compels an interpolating perfect spline to be 0 . M. then necessarily (5.1) prevails. We now proceed to elaborate pertinent facts.

Denote the knots of $P(x)$ by $\left\{\xi_{i}\right\}_{i=1}^{s}$ arranged in increasing order and assume $s \leqslant r$. Assume $\left\{x_{i}\right\}_{1}^{n+r+1}$ consist of distinct points. Denote by

$$
\begin{aligned}
& e_{i}^{(k)}=P\left(x_{i}, x_{i+1}, \cdots, x_{i+k}\right), \\
& \quad i=1,2, \cdots, n+r-k+1, k=0,1,2, \cdots, n-1,
\end{aligned}
$$

the $k$ th divided difference of $P$ with respect to the points $x_{i}, x_{i+1}, \cdots, x_{i+k}$. Manifestly, $e_{i}^{(k)}$ involve expressions only of the $\alpha_{i}$ and $x_{i}$ values. More specifically,

$$
\begin{aligned}
e_{i}^{(0)} & =P\left(x_{i}\right)=\alpha_{i}, \quad e_{i}^{(1)}=\frac{P\left(x_{i}\right)-P\left(x_{i+1}\right)}{x_{i}-x_{i+1}}=\frac{\alpha_{i}-\alpha_{i+1}}{x_{i}-x_{i+1}}, \\
e_{i}^{(2)} & =\left\{\frac{\left[P\left(x_{i}\right)-P\left(x_{i+1}\right)\right]}{x_{i}-x_{i+1}}-\frac{\left[P\left(x_{i+1}\right)-P\left(x_{i+2}\right)\right]}{x_{i+1}-x_{i+2}}\right\} \frac{1}{\left(x_{i}-x_{i+2}\right)} \\
& =\left\{\left(\frac{\alpha_{i}-\alpha_{i+1}}{x_{i}-x_{i+1}}-\frac{\alpha_{i+1}-\alpha_{i+2}}{x_{i+1}-x_{i+2}}\right)\right\} \frac{1}{x_{i}-x_{i+2}}
\end{aligned}
$$

etc. $\cdots$.

The next theorem locates the knots relative to the interpolating points provided the given interpolating data fulfills suitable oscillation characteristics.

THEOREM 5.1. Let $X=\left\{x_{i}\right\}_{1}^{n+r+1}$ be prescribed distinct interpolating points and $\Gamma=\left\{\alpha_{i}\right\}_{1}^{n+r+1}$ the corresponding given data. Let $P$ be a perfect spline (recall that $P$ is not necessarily uniquely determined; $\S 6$ will discuss this matter) interpolating the data $\Gamma$ at $X$ with corresponding knots $0<\xi_{1}<\xi_{2}<$ $\cdots<\xi_{s}<1(s \leqslant r)$. If for some $k \geqslant 0$

$$
e_{i}^{(k)} e_{i+1}^{(k)}<0, \quad i=1,2, \cdots, n-r-k,
$$

then necessarily $s=r$ and

$$
x_{i}<\xi_{i}<x_{i+n+1}, \quad i=1,2, \cdots, r .
$$

(Remark. In (5.4), $\leqslant 0$ suffices.) 
COROLlARY.5.1. Under the conditions of the theorem, the determinant

$\Phi\left(\begin{array}{c}x_{1}, x_{2}, \cdots, x_{n+2}, \cdots, x_{n+r+1} \\ 0, \cdots, 0, \xi_{1}, \cdots, \xi_{r}\end{array}\right)$

(5.6)

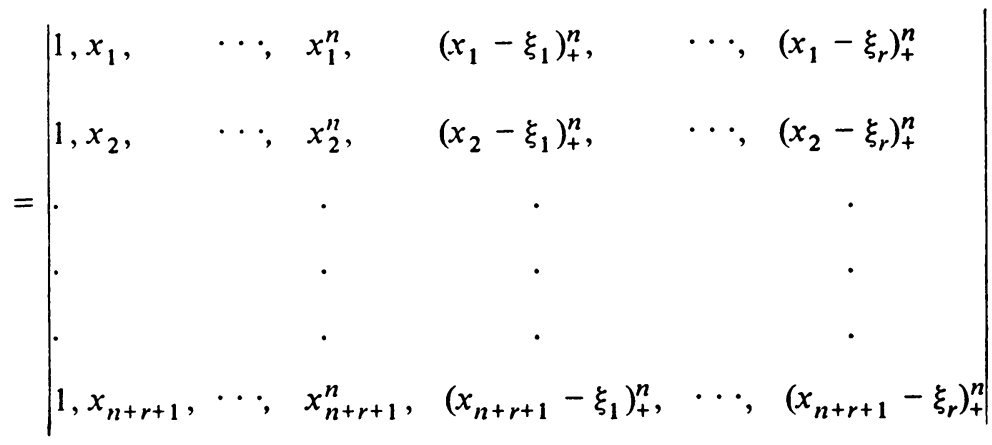

is positive.

Proof. The corollary follows from the known criteria when (5.6) is positive (see [3, p. 503]). For the case at hand the assertion (5.6) is equivalent to the requirements of (5.5).

We now turn to the proof of Theorem 5.1. It is instructive to treat first the case $k=0$. Assume therefore

$$
P\left(x_{i}\right) P\left(x_{i+1}\right)=e_{i}^{(0)} e_{i+1}^{(0)}<0, \quad i=1,2, \cdots, n+r .
$$

The hypothesis (5.4) when $k=0$ implies that $P(y)$ changes sign at least once in each interval $\left(x_{i}, x_{i+1}\right)$. Therefore, certainly $P\left(y_{i}\right)=0, x_{i}<y_{i}<x_{i+1}$, and $P$ changes sign at each $y_{i}, i=1,2, \cdots, n+r$. Now suppose to the contrary of (5.5) that for some $i_{0}, \xi_{i_{0}} \geqslant x_{n+i_{0}+1}$. Consider $P(x)$ restricted to $\left(0, \xi_{i_{0}}\right)$. Clearly $y_{n+i_{0}}<x_{n+i_{0}+1} \leqslant \xi_{i_{0}}$, so that $P$ vanishes at least $n+i_{0}$ times in (0, $\left.\xi_{i_{0}}\right)$. Successive differentiations and appeal to Rolle's theorem implies that $P^{(n-1)}(x)$ changes sign at least $i_{0}+1$ times on $\left(0, \xi_{i_{0}}\right)$. But $P^{(n-1)}(x)$ has the form $P^{(n-1)}(x)=\alpha+c\left[x-2\left(x-\xi_{1}\right)_{+}+2\left(\dot{x}-\xi_{2}\right)_{+}+\cdots+2(-1)^{i_{0}-1}\left(x-\xi_{i_{0}-1}\right)_{+}\right]$ on $\left(0, \xi_{i_{0}}\right)$ from which it is readily discerned that in this interval $P^{(n-1)}$ can change signs at most $i_{0}$ times. This contradiction can be averted only if $\xi_{i_{0}}<$ $x_{n+i_{0}+1}$ holds.

In a symmetrical manner we infer that if $\xi_{j_{0}} \leqslant x_{j_{0}}$, then $P(x)$ on $\left(\xi_{j_{0}}, \infty\right)$ exhibits at least $n+r+1-j_{0}$ changes of sign but involves only $r-j_{0}$ knots. We deduce a contradiction as previously so we must have $x_{j}<\xi_{j}, j=1,2, \cdots$, 
$r$. Theorem 5.1 is established under the hypothesis of (5.4) with $k=0$.

We turn now to the general case of (5.4) where $k>0$. The representation formula for the divided difference

$$
e_{i}^{(k)}=P\left(x_{i}, \cdots, x_{i+k}\right)=\frac{1}{k !} \int_{x_{i}}^{x_{i+k}} M_{i}(\xi) P^{(k)}(\xi) d \xi
$$

will be invoked (see [3, Chapter 10, (4.8)]). Here $M_{i}(\xi)$ constitutes a totally positive kernel with respect to the variables $0<\xi<\infty$ and $i=1,2,3, \cdots$ (see [3, p. 527]).

We can write

$$
e_{i}^{(k)}=\frac{1}{k !} \int_{x_{\nu}}^{x_{n+r+1}} M_{i}(\xi) P^{(k)}(\xi) d \xi, \quad i=\nu, \nu+1, \cdots, n+r-k,
$$

since $M_{i}(\xi)$ vanishes outside $\left(x_{i}, x_{i+k}\right)$. The hypothesis tells us that the sequence $e_{\nu}^{(k)}, e_{\nu+1}^{(k)}, \cdots, e_{n+r-k+1}^{(k)}, \nu, n>0$, displays $n+r-k-\nu+1$ changes of sign.

Appeal to the variation diminishing character of the kernel $M_{i}(\xi)$ implies that

$$
\begin{aligned}
& P^{(k)}(\xi) \text { changes sign at least } n+r-k-\nu+1 \text { times } \\
& \text { on the interval }\left(x_{\nu}, x_{n+r+1}\right) .
\end{aligned}
$$

Now suppose contrary to (5.5) that for some $\nu, \xi_{\nu} \leqslant x_{\nu}$ holds. Then (5.8) prevails. Successive application of Rolle's theorem yields that

$$
P^{(n-1)}(\xi)=\gamma+c\left[x+2 \sum_{\mu=\nu+1}^{r}(-1)^{\mu}\left(x-\xi_{\mu}\right)_{+}^{1}\right]
$$

changes sign at least $r-\nu+2$ on $\left(\xi_{\nu}, \infty\right)$. But this conclusion is absurd and therefore $x_{i}<\xi_{i}, i=1,2, \cdots, r$, must hold.

By analogous arguments exploiting the expression

$$
e_{i}^{(k)}=\frac{1}{k !} \int_{0}^{x_{\mu+n+1}} M_{i}(\xi) P^{(k)}(\xi) d \xi, \quad i=1,2, \cdots, \mu+n+1-k,
$$

we establish $\xi_{\mu}<x_{\mu+n+1}, \mu=1,2, \cdots, r$.

The proof of Theorem 5.1 is complete.

A sharper result than (5.5) can be achieved when the given data $\left\{\alpha_{i}\right\}$ is further specialized.

THEOREM 5.2. Let $\left\{x_{i}\right\}_{1}^{n+r+1}$ be given with corresponding data $\left\{\alpha_{i}\right\}_{1}^{n+r+1}$ where all $\alpha_{i}=0 \quad\left(i \neq i_{0}\right)$ except $\alpha_{i_{0}}=1$. Let $P$ be a perfect spline interpolating $\left\{\alpha_{i}\right\}$ at $\left\{x_{i}\right\}$ with knots $\left\{\xi_{i}\right\}_{1}^{s}(s \leqslant r)$. Then $s=r$ and we have

and

$$
x_{\nu+1}<\xi_{\nu} \text { for all } i_{0} \leqslant \nu
$$

$$
\xi_{\mu}<x_{\mu+n} \text { for all } \mu+n<i_{0} .
$$

Proof. Since $P$ vanishes at $n+r$ points we must have $s=r$ (see Lemma 
5.1 below). Suppose to the contrary of (5.10) that $\xi_{\nu} \leqslant x_{\nu+1}$ for some $\nu \geqslant$ $i_{0}$. Then on the interval $\left(\xi_{\nu}, \infty\right), P(x)$ vanishes $n+r-\nu+1$ times and involves precisely $r-\nu$ knots. It follows that $P^{(n-1)}(x)$ changes sign at least $r-\nu+2$ times on $\left(\xi_{\nu}, \infty\right)$ a result clearly incompatible with the existence of only $r-\nu$ knots.

The relation (5.11) is proved in a parallel manner to (5.10) concentrating attention on an interval of the type $\left(0, \xi_{\mu}\right)$.

LEMMA 5.1. Any nontrivial perfect spline with $r$ knots can only vanish at at most $n+r$ isolated points.

Proof. Since the derivative of a perfect spline remains a perfect spline of reduced degree a simple induction establishes the assertion of the lemma.

6. Criteria for uniqueness of interpolating perfect splines. For any real piecewise continuous function $f$ defined on $[a, b]$ let $S^{-}\{f(x) ;[a, b]\}$ denote the number of sign changes of $f$ as $x$ traverses the interval $a$ to $b$.

It is convenient to display the following elementary facts stated as the content of the next lemma.

Lemma 6.1. Let $P(x)$ and $Q(x)$ be two perfect splines of degree zero with $p$ and $q$ knots respectively, all located in $(0,1)$. Suppose $|P(x)| \equiv|Q(x)| \equiv c=$ constant.

(i) Then

$$
S^{-}\{P-Q ;[0,1]\} \leqslant \min (p, q) .
$$

(i') If $|P(x)|>|Q(x)|$ then the bound in (6.1) is $p$.

(ii) Suppose that $p=q, \operatorname{sign} P(0)=\operatorname{sign} Q(0)$. We then have

$$
S^{-}\{P-Q ;[0,1]\} \leqslant p-1,
$$

while if $\operatorname{sign} P(0)=-\operatorname{sign} Q(0)$, then

$$
S^{-}\{P-Q ;[0,1]\}=p
$$

occurs if the knots of $P$ and $Q$ strictly interlace. (See also Remark (6.1) below.)

Proof. (i) Let $\xi_{1}<\xi_{2}<\cdots<\xi_{p}$ and $\eta_{1}<\eta_{2}<\cdots<\eta_{q}$ denote the knots of $P$ and $Q$ respectively. The function $P$ of constant absolute value alternates between its maximum $c$ and its minimum $-c$ exclusively at the points $\left\{\xi_{i}\right\}_{1}^{p}$. It follows as long as $|Q(x)| \leqslant c$ for all $x$ that $S^{-}\{P-Q ;[0,1]\} \leqslant p$. Since $Q$ is also a perfect spline by a symmetrical argument we infer $S^{-}\{P-Q ;[0,1]\} \leqslant q$ and these inequalities in conjunction yield (6.1). 
(i') The proof goes analogously to (i).

(ii) Consider $P(0)=Q(0)=c$ and suppose at first the circumstance $\eta_{1} \geqslant$ $\xi_{1}$. A little reflection convinces one that $P(x)-Q(x)$ keeps one sign certainly over the stretch $0 \leqslant x \leqslant \xi_{2}$. Subsequent sign changes of $P-Q$ can arise only at the knot points $\xi_{2}, \xi_{3}, \cdots, \xi_{p}$ for the same reason as in case (i). Therefore, (6.2) obtains. Where $\eta_{1}<\xi_{1}$ takes place we arguc in a parallel fashion with reference to the knots $\left\{\eta_{2}, \cdots, \eta_{p}\right\}$. Thus in all events (6.2) is established.

If the knots $\left\{\xi_{i}\right\}_{1}^{p}$ and $\left\{\eta_{j}\right\}_{1}^{p}$ strictly interlace and $Q(0)=-P(0)$ then it is easy to check the equality of (6.3).

The proof of Lemma 6.1 is complete.

REMARK 6.1. The equation (6.3) can hold even where the knots of $P$ and $Q$ do not necessarily interlace. For example, if $P(0)=-Q(0)$ and $\eta_{1}<\xi_{1}<$ $\xi_{2}<\eta_{2}<\eta_{3}<\xi_{3}<\cdots<\xi_{p}<\eta_{p}$ ( $p$ odd) then (6.3) is maintained.

We are now prepared to present some conditions guaranteeing uniqueness of the interpolating spline whose existence was affirmed in Theorem 1. It is instructive and useful to first display two simple cases of nonuniqueness.

EXAMPLE 1. $n=0, r=3$.

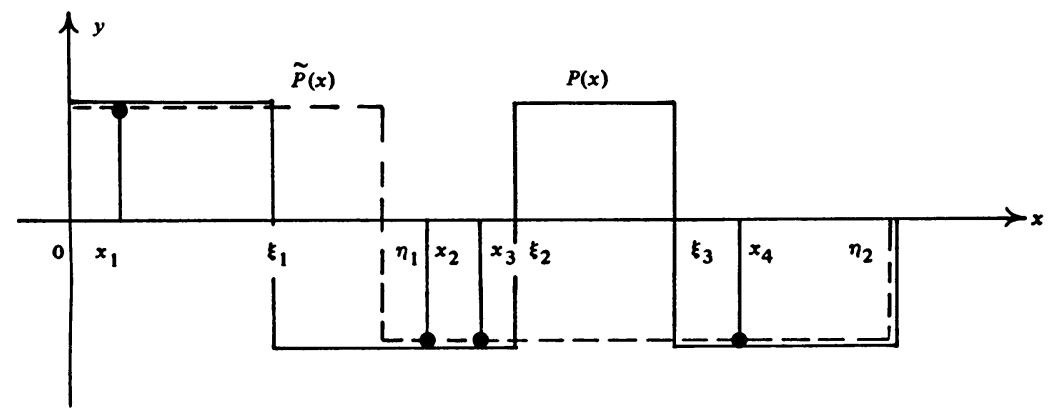

Clearly, $P(x)$ and $\widetilde{P}(x)$ are both solutions for the data at $\left\{x_{i}\right\}_{1}^{4}$.

EXAMPLE 2. $n=1, r=3$.

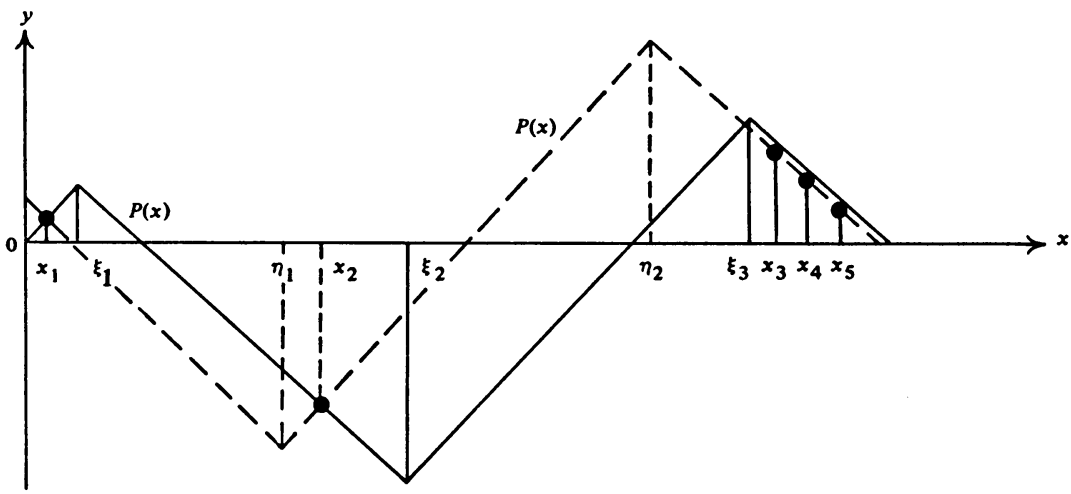


Again, $P(x)$ and $\widetilde{P}(x)$ are both solutions for the data at $\left\{x_{i}\right\}_{1}^{\mathbf{5}}$.

Theorems 6.1 and 6.2 below offer sufficient conditions for uniqueness of the interpolating perfect spline.

THEOREM 6.1. Let the interpolating points $\left\{x_{i}\right\}_{1}^{n+r+1}$ be given containing no block of coincident $x$ 's exceeding $n$ and corresponding prescribed real data $\left\{\alpha_{i}\right\}_{1}^{n+r+1}$ with $\Sigma \alpha_{i}^{2}>0$. Suppose the data implies for any interpolating spline $P(x)$ of degree $n$ with knots $\left\{\xi_{\nu}\right\}_{1}^{s}, s \leqslant r$, the relations

$$
x_{\nu}<\xi_{\nu}<x_{\nu+n+1}, \quad \nu=1,2, \cdots, s,
$$

(see Theorem 5.1 for conditions ensuring that (6.4) holds). Provided $r \leqslant n$, the interpolating spline is unique.

Proof. Let $P(x)$ and $Q(x)$ be interpolating perfect splines of degree $n$ each involving at most $r$ knots.

Suppose $P(x)$ has knots $0<\xi_{1}<\cdots<\xi_{s_{1}}<1, s_{1} \leqslant r$, and $Q(x)$ has knots $0<\eta_{1}<\cdots<\eta_{s_{2}}<1, s_{2} \leqslant r$.

Define $h(x)=P(x)-Q(x)$. We must prove $h(x) \equiv 0$ for $x \in[0,1]$.

Case 1. Suppose $h(x)$ is not identically zero on any interval (of positive measure) $\subseteq[0,1]$. Then $h\left(x_{i}\right)=P\left(x_{i}\right)-Q\left(x_{i}\right)=0, i=1, \cdots, n+r+1$. Invoking repeatedly Rolle's theorem, it follows that $h^{(n)}(x)$ admits at least $r+1$ sign changes in $(0,1)$. Note that $\left|P^{(n)}(x)\right|$ and $\left|Q^{(n)}(x)\right|$ are constant functions. Suppose $\left|P^{(n)}(x)\right| \geqslant\left|Q^{(n)}(x)\right|$. But $P^{(n)}(x)$ has $s_{1} \leqslant r$ knots and hence $h^{(n)}(x)$ $=P^{(n)}(x)-Q^{(n)}(x)$ exhibits at most $s_{1} \leqslant r$ sign changes (cf. the proof of Lemma 6.1), a contradiction. Therefore Case 1 cannot happen.

Case 2. Consider the possibility that $h(x)$ vanishes identically on some interval $\subseteq[0,1]$. Then on that interval $P^{(n)}(x) \equiv Q^{(n)}(x)$. But $\left|P^{(n)}(x)\right|$ and $\left|Q^{(n)}(x)\right|$ being constant throughout $[0,1]$, it follows that $\left|P^{(n)}(x)\right| \equiv\left|Q^{(n)}(x)\right|$ $\equiv c$ for all $x \in[0,1]$. It is clear that $h^{(n)}(x)$ is a step function whose only possible values are $-2 c, 0,+2 c$. It has jumps possible only at the knots $\xi_{i}$ of $P(x)$ and $\eta_{i}$ of $Q(x)$. We shall distinguish three contingencies:

Case 2.1. $h(x) \neq 0$ in a neighborhood of the endpoint 0 but $h(x)$ vanishes in some interval.

Case 2.2. $h(x) \neq 0$ in a neighborhood of 1 but vanishes in some interval.

Case 2.3. $h(x) \equiv 0$ in a neighborhood of 0 and 1 but $h(x)$ is not identically zero throughout $[0,1]$.

Case 2.1. The initial interval on which $h \equiv 0$ commences at some $\xi_{\nu}$ or $\eta_{\mu}$. Suppose $h(x)=P(x)-Q(x) \equiv 0$ for $x \in\left[\xi_{\nu}, \xi_{\nu}+\epsilon\right), \epsilon>0$, where $\xi_{\nu}+\epsilon$ is either $\xi_{\nu+1}$ or $\min _{\mu}\left\{\eta_{\mu} \mid \eta_{\mu}>\xi_{\nu}\right\}$, or 1 . Because $P(x), Q(x) \in W_{\infty}^{(n)}$ and 
$P(x) \equiv Q(x)$ on $\left[\xi_{\nu}, \xi_{\nu}+\epsilon\right)$, we have $h^{(i)}\left(\xi_{\nu}\right)=0, i=0, \cdots, n-1$. Concentrate now on $\left[0, \xi_{\nu}\right]$. Because of the relations $x_{j}<\xi_{j}<x_{j+n+1}, j=1, \cdots$, $s_{1} \leqslant r$, we have that $x_{1}, \cdots, x_{\nu} \in\left[0, \xi_{\nu}\right)$ and $h\left(x_{j}\right)=0, j=1, \cdots, \nu$. (The coincidence of two $x$ 's is interpreted as $h\left(x_{i}\right)=h^{\prime}\left(x_{i}\right)=0$ and similarly for higher order coincidences.) In $\left[0, \xi_{\nu}\right]$ we will invoke repeatedly Rolle's theorem and also use the fact that $h(x)$ does not vanish identically on any subinterval of $\left[0, \xi_{\nu}\right]$. Certainly $h(x)$ vanishes at $x_{1}, \cdots, x_{\nu}$ and at $\xi_{\nu}$. By virtue of Rolle's theorem we infer that $h^{\prime}(x)$ displays at least $\nu$ zeros in $\left(0, \xi_{\nu}\right)$ and also we have $h^{\prime}\left(\xi_{\nu}\right)=0$.

Repeating the same argument several times we deduce the fact that $h^{(n-1)}(x)$ admits at least $\nu$ isolated zeros in $\left(0, \xi_{\nu}\right)$, and also $h^{(n-1)}\left(\xi_{\nu}\right)=0$ prevails. Another application of Rolle's theorem implies that $h^{(n)}(x)$ exhibits at least $\nu$ sign changes in $\left[0, \xi_{\nu}\right)$.

On the other hand, $h^{(n)}(x)=P^{(n)}(x)-Q^{(n)}(x)$ is a difference of two zeroth degree perfect splines where $P^{(n)}$ involves precisely $\nu-1$ knots situated in $\left[0, \xi_{\nu}\right)$ and $\left|P^{(n)}(x)\right| \equiv\left|Q^{(n)}(x)\right|$ holds. On account of (6.1) it follows that $S^{-}\left\{h^{(n)}(x) ;\left[0, \xi_{\nu}\right]\right\} \leqslant \nu-1$, which is incompatible with the last comment of the preceding paragraph. The only tenable conclusion is that Case 2.1 is not possible.

Case 2.2. The analysis paraphrases that of Case 2.1 where the role of the interval $\left[0, \xi_{v}\right)$ is replaced by a suitable subinterval extending to the endpoint 1 .

Case 2.3. We suppose that $h(x) \equiv 0$ in a neighborhood of zero and one. We will arrive at a contradiction unless $P(x) \equiv Q(x)$ holds throughout $[0,1]$. There are essentially three subcases indicating the contrary possibilities.

Subcase 2.3.1. There exists an internal interval $\left(\xi_{\nu}, \xi_{\mu}\right), \nu<\mu$ where $h(x) \equiv 0$ in any subinterval of positive length but $h(x) \equiv 0$ on $\left(\xi_{\nu}-\epsilon, \xi_{\nu}\right]$ and on $\left[\xi_{\mu}, \xi_{\mu}+\epsilon\right)$ for some $\epsilon>0$.

Subcase 2.3.2. The conditions analogous to Subcase 2.3.1 are present where $\xi_{\nu}$ and $\xi_{\mu}$ are replaced by $\eta_{\nu}$ and $\eta_{\mu}$ respectively.

Subcase 2.3.3. $h(x) \equiv 0$ on $\left(\xi_{\nu}-\epsilon, \xi_{\nu}\right]$ and on $\left[\eta_{\mu}, \eta_{\mu}+\epsilon\right), \xi_{\nu}<\eta_{\mu}$ (or the symmetric case with $\eta_{\nu}$ and $\xi_{\mu}$ ). Moreover, $h(x) \neq 0$ in any subinterval of positive length of $\left(\xi_{\nu}, \eta_{\mu}\right)$.

Subcase 2.3.1. Concentrate on the interval $\left[\xi_{\nu}, \xi_{\mu}\right]$ and note the following facts:

(i) $P$ involves at most $\mu-\nu-1$ knots.

(ii) $h^{(i)}\left(\xi_{\nu}\right)=h^{(i)}\left(\xi_{\mu}\right)=0, i=0,1, \cdots, n-1$.

Suppose at first that

$$
\mu-\nu \leqslant n .
$$

Repeated application of Rolle's theorem in view of (ii) implies that $h^{(n)}(x)$ exhibits at least $n$ sign changes in $\left(\xi_{\nu}, \xi_{\mu}\right)$ and this fact violates property (i) (in view of relation (6.2)). 
Suppose next that

$$
\mu-\nu>n, \text { say } \mu-\nu=n+k, k \geqslant 1 .
$$

In the presence of (6.7) we claim that at least $k$ of the $x_{\sigma}$ 's are contained in $\left(\xi_{\nu}, \xi_{\mu}\right)$. Because of the relations (6.4), we have

and the claim is substantiated.

$$
\xi_{\nu}<x_{n+\nu+1} \leqslant x_{n+\nu+k}<\xi_{n+\nu+k}=\xi_{\mu},
$$

Thus in addition to the multiple vanishing at $\xi_{\nu}$ and $\xi_{\mu}$ as indicated in (ii) above, $h(x)$ also vanishes at at least $k$ internal points of $\left(\xi_{\nu}, \xi_{\mu}\right)$. Invoking Rolle's theorem repeatedly leads to the inference that $h^{(n)}(x)$ exhibits at least $k+n$ sign changes in $\left(\xi_{\nu}, \xi_{\mu}\right)$. But $P(x)$ in $\left(\xi_{\nu}, \xi_{\mu}\right)$ includes exactly $\mu-\nu-$ $1=n+k-1$ knots and a contradication ensues as in the previous cases. The fact that the Subcase 2.3.1 cannot occur is thus established independent of the value of $r$.

Subcase 2.3.2. This situation is precluded by entirely analogous deliberations as in the preceding case.

Subcase 2.3.3. Recall that here $h(x) \equiv 0$ on $\left(\xi_{\nu}-\epsilon, \xi_{\nu}\right]$ and on $\left[\eta_{\mu}, \eta_{\mu}+\right.$ $\epsilon)$ but $h(x) \neq 0$ on any subinterval of $\left(\xi_{\nu}, \eta_{\mu}\right)$.

We examine three possibilities:

(i) $\eta_{\mu} \leqslant \xi_{\mu}$; here we paraphrase the analysis of Subcase 2.3.1. and a contradiction ensues.

(ii) $\eta_{\nu} \leqslant \xi_{\nu}$; a contradiction results as in Subcase 2.3.2.

(iii) It remains to consider the circumstance of $\xi_{\nu}<\eta_{\nu} ; \xi_{\mu}<\eta_{\mu}$.

We now make the following assertion. $\left({ }^{2}\right) P$ and $Q$ include an equal number of knots inside $\left(\xi_{\nu}, \eta_{\mu}\right)$. To validate this statement, let $\eta_{k}$ be the smallest knot of $Q$ exceeding $\xi_{\nu}$ and denote by $\xi_{l}$ the largest knot of $P$ less than $\eta_{\mu}$. Thus, the knots of $P$ in $\left(\xi_{\nu}, \eta_{\mu}\right)$ are $\xi_{\nu+1}, \cdots, \xi_{l}$, their number $l-\nu$. The $\eta_{i}$ knots in $\left(\xi_{\nu}, \eta_{\mu}\right)$ are $\eta_{k}, \cdots, \eta_{\mu-1}$, and their number $\mu-k$. Suppose $l-$ $\nu \neq \mu-k$ and without loss of generality take $\mu-k>l-\nu$. Suppose first that $l-\nu \leqslant n-1$. Consider $h(x)$ in $\left(\xi_{\nu}, \eta_{\mu}\right)$. By Lemma 6.1, we have

$$
S^{-}\left\{h^{(n)}(x) ;\left(\xi_{\nu}, \eta_{\mu}\right)\right\} \leqslant \min (l-\nu, \mu-k)=l-\nu \leqslant n-1 .
$$

On the other hand in the usual way (since $h^{(i)}\left(\xi_{\nu}\right)=h^{(i)}\left(\eta_{\mu}\right)=0, i=0,1, \cdots$, $n-1)$, we deduce that $h^{(n)}(x)$ displays at least $n$ sign changes in $\left(\xi_{\nu}, \eta_{\mu}\right)$ and an absurdity results. Suppose next that $l-\nu \geqslant n$. We have $\mu-k>l-\nu=$ $n+\gamma, \gamma \geqslant 0$ a nonnegative integer. Hence $\mu>k+n+\gamma$. Referring to (6.4) we find the following inequalities hold

$$
\xi_{\nu}<\eta_{k}<x_{n+1+k} \leqslant x_{n+2+k} \leqslant \cdots \leqslant x_{n+\gamma+k} \leqslant x_{\mu}<\eta_{\mu} .
$$

(2) The following argument is not needed to finish the proof but is helpful in the next theorem. 
Hence $h(x)$ possesses at least $\mu-n-k$ zeros in $\left(\xi_{\nu}, \eta_{\mu}\right)$ and of course, $h^{(i)}\left(\xi_{\nu}\right)$ $=h^{(i)}\left(\eta_{\mu}\right)=0, i=0,1, \cdots ; n-1$. It follows that $h^{(n)}(x)$ exhibits in $\left(\xi_{\nu}, \eta_{\mu}\right)$ at least $\mu-k \geqslant l-\nu+1$ sign changes. But $S^{-1}\left\{h^{(n)}(x) ;\left(\xi_{\nu}, \eta_{\mu}\right)\right\} \leqslant$ $l-\nu$, and a contradiction is apparent. Therefore, we have necessarily $\mu-k=$ $l-\nu$. The assertion is proved.

Suppose $r \leqslant n$. So $P$ and $Q$ both have at most $r-1$ knots in $\left(\xi_{\nu}, \eta_{\mu}\right)$. Referring to (6.2) we have

$$
S^{-}\left\{h^{(n)}(x) ;\left(\xi_{\nu}, \eta_{\mu}\right)\right\} \leqslant n-1 .
$$

But, on the other hand $h^{(i)}\left(\xi_{\nu}\right)=h^{(i)}\left(\eta_{\mu}\right)=0, i=0, \cdots, n-1$, implies that

$$
h^{(n)}(x) \text { has at least } n \text { sign changes in }\left(\xi_{\nu}, \eta_{\mu}\right) \text {, }
$$

and (6.8) and (6.9) are incompatible. We covered all the possibilities always producing a contradiction. It follows that $h(x) \equiv 0$ on $[0,1]$, and the proof of Theorem 6.1 is complete.

REMARK 6.2. The only way in which nonuniqueness can occur for $r>n$ corresponds to Subcase 2.3.3. The examples of nonuniqueness depicted next maintain the relations (6.4) but the requirement $r \leqslant n$, of course, fails.

EXAMPLE 3. $n=1, r=4, c=1$.

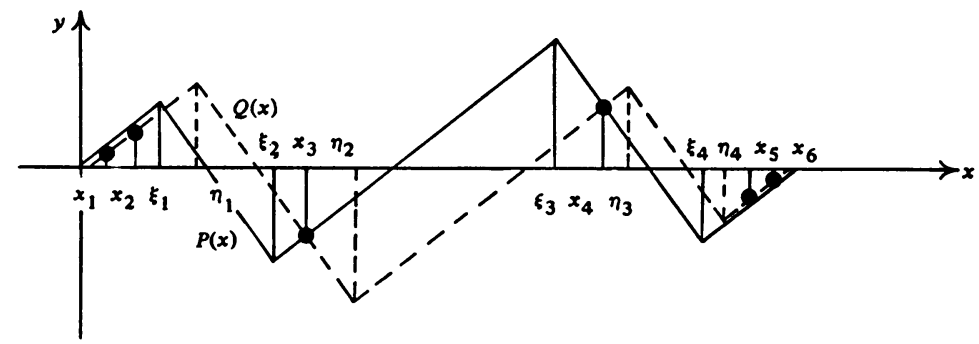

The conditions for the knots (6.4) are satisfied.

EXAMPLE 4. $n=1, r=3, c=1$.

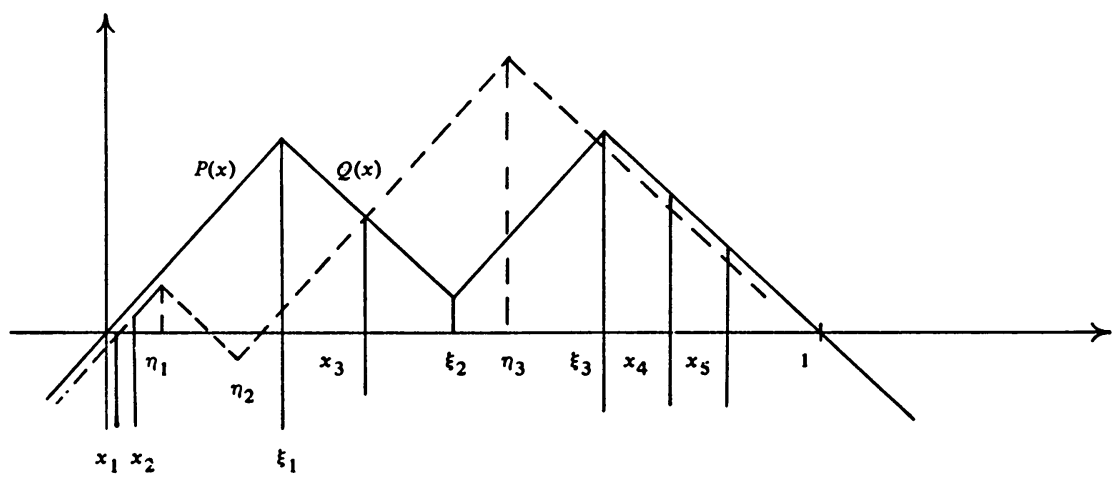

The conditions (6.4) are again met. 
EXAMPLE 5. $n=1, r=2, c=1$.

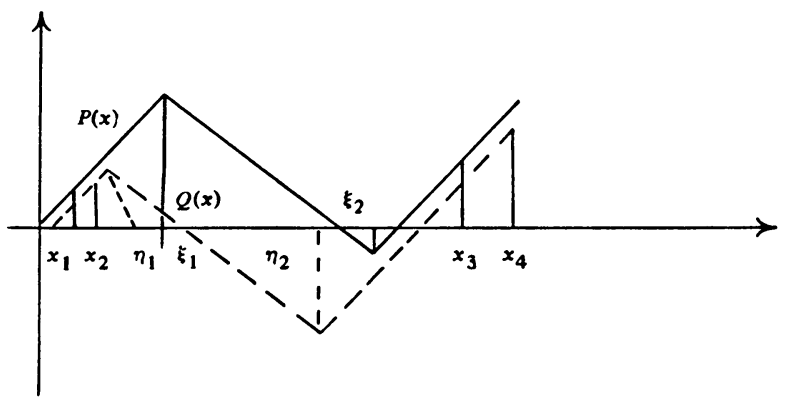

Notice in Example 3 that the knots of the two splines interlace

$$
\xi_{1}<\eta_{1}<\xi_{2}<\eta_{2}<\xi_{3}<\eta_{3}
$$

while in Example 4 they have the order

$$
\eta_{1}<\eta_{2}<\xi_{1}<\xi_{2}<\eta_{3}<\xi_{3} .
$$

Example 5 shows that $r=n$ is sharp.

If the conditions (6.4) are not satisfied then even with $r \leqslant n$ nonuniqueness can hold. Examples of this kind are easily constructed. However, where $r \leqslant n$ the perfect interpolating spline with the minimal number of knots is unique within the class of the competing interpolating splines of the same number of knots.

The following corollary affirms uniqueness in the special case Glaesser examined ([2], see also the introduction).

COROLlaRY 6.1. Let $r=n-1$. Let the interpolation points be $\left\{x_{i}\right\}_{1}^{2 n}$ where $x_{1}=x_{2}=\cdots=x_{n}=0, x_{n+1}=\cdots=x_{2 n}=1$, and with associated data $\left\{\alpha_{i}\right\}_{1}^{2 n}, \Sigma \alpha_{i}^{2}>0$. The interpolating perfect spline is uniquely determined.

Proof. It is only necessary to check that the conditions (6.4) are satisfied. This is immediate.

The next theorem gives a case of uniqueness valid for all $r$. This result will serve fundamentally in our developments pertaining to Theorems 5-7 of the introduction.

THEOREM 6.2. Suppose $\left\{x_{i}\right\}_{1}^{n+r+1}$ are given containing no coincident block exceeding $n$ points. Let the associated data $\left\{\alpha_{i}\right\}_{1}^{n+r+1}$ be such that

$$
\alpha_{i}=0 \cdot \text { for all } i \neq i_{0} ; \quad \alpha_{i_{0}} \neq 0 \text {. }
$$

(All data except one have zero value.) Then the interpolating perfect spline of degree $n$ with at most $r$ knots is unique. (Emphasis: Here $r$ is any positive integer.)

REMARK 6.3. Recall from Theorem 5.2 that any interpolating spline for the 
data (6.10) necessarily involves $r$ knots and the relations of the knots and interpolatory points satisfy

$$
\begin{aligned}
x_{\nu+1}<\xi_{\nu} & \text { for all } \nu \geqslant i_{0}, \\
\xi_{\mu}<x_{\mu+n} & \text { for all } \mu+n \leqslant i_{0} .
\end{aligned}
$$

PROoF. Scrutiny of the proof of Theorem 6.1 reveals that only Subcase 2.3.3 need be examined (see also Remark 6.2). Thus assume $h(x)=P(x)-Q(x)$ vanishes on $\left(\xi_{\nu}-\epsilon, \xi_{\nu}\right)$ and on $\left(\eta_{\mu}, \eta_{\mu}+\epsilon\right)$ for some $\epsilon>0$ and $h(x)$ vanishes on no subinterval of $\left(\xi_{\nu}, \eta_{\mu}\right)$. We proved during the analysis of Subcase 2.3.3 that $P$ and $Q$ have the same number of knots $\left\{\xi_{\nu+1}, \cdots, \xi_{l}\right\}$ and $\left\{\eta_{k}\right.$, $\left.\cdots, \eta_{\mu-1}\right\}$ located in $\left(\xi_{\nu}, \eta_{\mu}\right)$ so that $l-\nu=\mu-k$. Suppose

$$
\mu-k=l-\nu=n+\gamma,
$$

with $\gamma$ a nonnegative integer. Both the $\xi$ knots and the $\eta$ knots satisfy (6.10) as attested to by Theorem 5.2. Consider three cases:

Case $1 . i_{0} \leqslant l$. By virtue of $(6.10)$ we have $x_{l+1}<\xi_{l}<\eta_{\mu}$ while $\xi_{\nu}<$ $x_{n+\nu+1}$. It is easy to see on the basis of (6.11) that $l+1 \geqslant n+k+1$ and therefore the number of $x$ 's inside $\left(\xi_{\nu}, \eta_{\mu}\right)$ is at least $l-\nu-n+1=\mu-n-$ $k+1$ one more than occurred previously in the analysis of Theorem 6.1, Case 2.3.3.

Now consider $h(x)=P(x)-Q(x)$. Note that $h^{(i)}\left(\xi_{\nu}\right)=h^{(i)}\left(\eta_{\mu}\right)=0, i=$ $0,1, \cdots, n-1$, and $h\left(x_{\sigma}\right)=0$ for all $x_{\sigma} \in\left(\xi_{\nu}, \eta_{\mu}\right)$. Implementing Rolle's theorem $n$ times leads to the fact that $h^{(n)}$ displays at least $\mu-k+1$ sign changes. On the other hand $P$ and $Q$ each involves $\mu-k$ knots in $\left(\xi_{\nu}, \eta_{\mu}\right)$ so that $h^{(n)}$ can change sign no more than $\mu-k$ times. This contradiction is only averted if $h(x) \equiv 0$.

Case 2. $i_{0} \geqslant k$. Now, we have because of (6.10)

$$
\eta_{k}<x_{k+n} \leqslant \cdots \leqslant x_{\mu}<\eta_{\mu}
$$

and again we find at least $\mu-k-n+1 x$ 's in $\left(\xi_{\nu}, \eta_{\mu}\right)$.

The argument is finished as in Case 1.

Case 3. $l<i_{0}<k$. It follows from (6.10) that $x_{\mu+1}<\eta_{\mu}$ and we also have $\eta_{k}<x_{k+n}$ implying the existence of at least $\mu-k-n+1 x$ 's inside $\left(\xi_{\nu}, \eta_{\mu}\right)$. Hereafter the proof is completed as in Case 1 .

7. The existence of some special perfect spline polynomials satisfying moment conditions. The variational problem (1.5b) admits an elegant solution when cast in terms of functions comprising a complete Tchebycheff system (C.T.S.).

Let $w_{1}(x), w_{2}(x), \cdots, w_{t+1}(x), t \geqslant n$, constitute a C.T.S. on the interval $(0,1)$. This property requires that the determinants 


$$
\epsilon_{p}\left|\begin{array}{cccc}
w_{1}\left(\eta_{1}\right) & w_{1}\left(\eta_{2}\right) & \cdots & w_{1}\left(\eta_{p}\right) \\
w_{2}\left(\eta_{1}\right) & w_{2}\left(\eta_{2}\right) & \cdots & w_{2}\left(\eta_{p}\right) \\
\cdot & \cdot & & \cdot \\
\cdot & \cdot & & \cdot \\
w_{p}\left(\eta_{1}\right) & w_{p}\left(\eta_{2}\right) & \cdots & w_{p}\left(\eta_{p}\right)
\end{array}\right|
$$

for all choices of $0<\eta_{1}<\eta_{2}<\cdots<\eta_{p}<1$ and $1 \leqslant p \leqslant t+1$ are strictly positive where $\epsilon_{p}$ is an appropriate sequence of \pm 1 signs independent of the $\left\{\eta_{i}\right\}$ (actually it suffices to require that the determinants are almost everywhere positive). The principal prototype of a C.T.S. are the monomials $w_{k}(x)=x^{k-1}$, $k=1,2, \cdots, t+1$.

Consider the collection of all $f \in W_{\infty}^{(n)}(0,1)$, the usual Sobolev space stipulating that $f^{(n-1)}$ is of Lipschitz class on $[0,1]$, satisfying the additional moment conditions

$$
\int w_{k}(x) f(x) d x=d_{k}, \quad k=1,2, \cdots, t+1,
$$

where $d_{k}$ are prescribed real constants not all zero.

Call this class $W_{\infty}^{n}\left(w_{1}, w_{2}, \cdots, w_{t+1}, \mathrm{~d}\right)=W^{(n)}(\mathrm{w}, \mathrm{d})$. The construction affirmed in Theorem 7.1 below will be basic for the solution of the following problem.

Characterize $f^{*} \in W_{\infty}^{(n)}(\mathbf{w}, \mathrm{d})$ which attains

$$
\min _{f \in W_{\infty}^{(n)}(w, d)}\left\|f^{(n)}\right\|_{\infty} .
$$

THEOREM 7.1. There exists a unique perfect spline polynomial exhibiting at most $t-n$ knots fulfilling the generalized moment conditions (7.2).

Proof. We will invoke Theorem 4 (see the introduction) with the specifications

$$
A_{k i}=\int_{0}^{1} w_{k}(x) x^{i-1} d x, \quad i=1,2, \cdots, n, k=1,2, \cdots, t+1,
$$

and

$$
u_{k}(\xi)=\int_{0}^{1} w_{k}(x)(x-\xi)_{+}^{n} d x, \quad k=1,2, \cdots, t+1,0 \leqslant \xi \leqslant 1 .
$$

Obviously,

$$
\frac{d u_{k}(\xi)}{d \xi}=v_{k}(\xi)=-n \int_{0}^{1} w_{k}(x)(x-\xi)_{+}^{n-1} d x .
$$

The corresponding determinant (1.7) for the case at hand (we use the composition formula of [3, p. 17], cf. equation (4.6)) reduces to (for $0<\eta_{1}<$ $\left.\eta_{2}<\cdots<\eta_{s}<1, n+s \leqslant t+1\right)$ 


$$
\left|\begin{array}{ccccc}
A_{11}, & \cdots, & A_{1, n}, v_{1}\left(\eta_{1}\right), & \cdots, & v_{1}\left(\eta_{s}\right) \\
A_{21}, & \cdots, & A_{2, n}, v_{2}\left(\eta_{1}\right), & \cdots, & v_{2}\left(\eta_{s}\right) \\
\cdot & \cdot & \cdot & & \cdot \\
\cdot & \cdot & \cdot & & \cdot \\
A_{n+s, 1}, & \cdots, & A_{n+s, n}, v_{n+s}\left(\eta_{1}\right), & \cdots, & v_{n+s}\left(\eta_{s}\right)
\end{array}\right|
$$$$
=(-n)^{s} \int_{0<x_{1}<x_{2}<\cdots<x_{n+s}<1}\left|\begin{array}{ccc}
w_{1}\left(x_{1}\right), & \cdots & w_{1}\left(x_{n+s}\right) \\
w_{2}\left(x_{1}\right), & \cdots & w_{2}\left(x_{n+s}\right) \\
\cdot & & \cdot \\
\cdot & & \cdot \\
\cdot & & \cdot \\
w_{n+s}\left(x_{1}\right), & \cdots & w_{n+s}\left(x_{n+s}\right)
\end{array}\right|
$$

$$
\times\left|\begin{array}{lll}
x_{1}^{0}, & \cdots & x_{n+s}^{0} \\
x_{1}^{1}, & \cdots & x_{n+s}^{1} \\
\cdot & & \cdot \\
x_{1}^{n-1}, & \cdots & x_{n+s}^{n-1} \\
\left(x_{1}-\eta_{1}\right)_{+}^{n-1}, & \cdots & \left(x_{n+s}-\eta_{1}\right)_{+}^{n-1} \\
\cdot & & \cdot \\
\left(x_{1}-\eta_{s}\right)_{+}^{n-1} & \cdots & \left(x_{n+s}-\eta_{s}\right)_{+}^{n-1}
\end{array}\right| d x_{1} d x_{2} \cdots d x_{n+s}
$$

The first determinant in the integrand maintains a strict sign in view of the hypothesis (7.1). The second determinant was encountered in the discussion of $\S 4$ where it was pointed out that the kernel $(x-\eta)_{+}^{n-1}$ is appropriately totally positive. It follows that condition (3.1) is met and Theorem 4 is applicable. Accordingly, let $\left\{\mathrm{a}^{*}, c^{*}, \xi^{*}\right\}$ satisfy

$$
\begin{array}{r}
\sum_{i=1}^{n} A_{k i} a_{i}^{*}+c^{*}\left[u_{k}(0)-2 u_{k}\left(\xi_{1}^{*}\right)+2 u_{k}\left(\xi_{2}^{*}\right)+\cdots+2(-1)^{s} u_{k}\left(\xi_{s}^{*}\right)\right] \\
=d_{k}, \quad k=1,2, \cdots, t+1 .
\end{array}
$$

Form the perfect spline

$$
P^{*}(x)=\sum_{i=1}^{n} a_{i}^{*} x^{i-1}+c^{*}\left[x^{n}-2\left(x-\xi_{1}^{*}\right)_{+}^{n}+\cdots+2(-1)^{s}\left(x-\xi_{s}^{*}\right)_{+}^{n}\right]
$$

Direct substitution for $P^{*}$ reveals that the equations

$$
\int_{0}^{1} P^{*}(x) w_{k}(x) d x=d_{k}, \quad k=1,2, \cdots, t+1,
$$

reduces to (7.6). 
The existence of a perfect spline $P^{*}$ in $W_{\infty}^{(n)}(\mathrm{w}, \mathrm{d})$ exhibiting at most $t-n$ knots is assured. It remains to demonstrate uniqueness.

Let $f$ be any member of $W_{\infty}^{(n)}(\mathrm{w}, \mathrm{d})$ distinct from $P^{*}$. Then

$$
\int_{0}^{1} w_{k}(x)\left(f(x)-P^{*}(x)\right) d x=0, \quad k=1,2, \cdots, t+1,
$$

prevails. Since $w_{k}$ constitute a complete Tchebycheff system we infer that $f-$ $P^{*}$ changes sign at least $t+1$ times (cf. Lemma 2.5). Repeated appeal to Rolle's theorem guarantees that $f^{(n)}-P^{*}(n)$ changes sign at least $t+1-n$ times. Where $f=Q$ is also a perfect spline in $W_{\infty}^{(n)}(\mathrm{w}, \mathrm{d})$, say involving $r$ knots $(r \leqslant$ $t-n$ ), then reference to Lemma 6.1 reveals that the number of sign changes of $Q^{(n)}-P^{(n)}$ cannot exceed $\min (t-n, r)$ and this statement is incompatible with our previous inferences. Therefore, the uniqueness assertion of Theorem 7.1 is established and the proof is complete.

For the special case where $w_{k}(x)=x^{k}, k=0,1, \cdots, t$, the existence and uniqueness of a perfect spline with at most $t-n$ knots satisfying the moment conditions (7.2) can be reduced to endpoint interpolation requirements. For this purpose, it is more convenient (and no restriction whatsoever) to write the moment conditions in the form

$$
\int_{0}^{1} w_{k}(x) f(x) d x=\int_{0}^{1}(1-x)^{k} f(x) d x=d_{k}, \quad k=0,1, \cdots, t .
$$

Now determine $g$ as a perfect spline of degree $n+t+1$ satisfying the interpolation condition

$$
g^{(j)}(1)=d_{t-j} /(t-j) !, \quad g^{(j)}(0)=0, j=0,1, \cdots, t .
$$

The existence and uniqueness of a spline with at most $t-n$ knots is assured by Theorem 1 and Corollary 6.1. Define

$$
f^{*}(x)=g^{(t+1)}(x) \text {. }
$$

Manifestly, $f(x)$ is a perfect spline of degree $n$ exhibiting the same knots as $g(x)$. We now verify that $f^{*}(x)$ satisfies the requisite moment condition. To this end, consider

$$
\int_{0}^{1} f^{*}(x)(1-x)^{i} d x=\int_{0}^{1} g^{(t+1)}(x)(1-x)^{i} d x
$$

and integrate by parts $i$ times with reference to (7.7) yielding

$$
\begin{array}{r}
\int_{0}^{1} f^{*}(x)(1-x)^{i} d x=i ! \int_{0}^{1} g^{(t+1-i)}(x) d x=i ! g^{(t-i)}(1)=d_{i}, \\
i=0,1,2, \cdots, t .
\end{array}
$$


8. Solutions of some variational problems by perfect splines. We are now prepared to present solutions of problems (1.3), (1.5a) and (1.5b) set forth in the introductory section.

THEOREM 8.1. Let $F$ be the subset of the Sobolev space $W^{(n)}[0,1]$ defined by

$$
F=W_{\infty}^{(n)}[0,1] \cap\left\{f \mid f\left(x_{i}\right)=\alpha_{i}, i=1,2, \cdots, n+r+1\right\},
$$

where $\left\{x_{i}\right\}$ and $\left\{\alpha_{i}\right\}$ are prescribed as in Theorem 1. Then any perfect spline $P$ in $F$ involving at most $r$ knots whose existence was confirmed in Theorem 1 satisfies

$$
\left\|P^{(n)}\right\| \leqslant\left\|f^{(n)}\right\| \quad\left(\|\cdot\| \text { is the usual } L^{\infty} \text { norm }\right)
$$

for all $f$ in $F$.

REMARK 8.1. The minimizing element of $F$ is not necessarily unique.

Proof. Let $P$ be a perfect spline in $F$. Suppose to the contrary of (8.1) that $f$ in $F$ satisfies

$$
\left\|f^{(n)}\right\|<\left\|p^{(n)}\right\| .
$$

Let $g=P-f$. In view of $(8.2)$ and since $\left|P^{(n)}(x)\right| \equiv c$ we infer that $g$ can vanish only at isolated points in $[0,1]$ which certainly must include the points $\left\{x_{i}\right\}_{i=1}^{n+r+1}$. Applying repeatedly Rolle's theorem we deduce that $g^{(n)}$ changes sign at least $r+1$ times on $[0,1]$. But $\left|P^{(n)}(x)\right| \equiv c$ and $P^{(n)}(x)$ changes values from $c$ to $-c$ exclusively at its knots. For the circumstance $\left\|f^{(n)}\right\|<\left\|P^{(n)}\right\|=c$, it would follow that $g^{(n)}$ changes sign at most $r$ times, an absurdity. The proof is complete.

Some further solutions of variational problems are now developed. Let $d_{i}$, $i=1,2, \cdots, t+1$, be given real numbers $t \geqslant n$ not all zero and let $\left\{w_{i}(x)\right\}_{1}^{t+1}$ be a C.T.S. as described in $\$ 7$. Define

$$
W_{\infty}^{(n)}(\mathrm{w}, \mathrm{d})=\left\{f \mid f \in W_{\infty}^{n}, \int_{0}^{1} f(x) w_{k}(x) d x=d_{k}, k=1,2, \cdots, t+1\right\} .
$$

Problem I. Find and characterize $f \in W_{\infty}^{(n)}(w, d)$ attaining

$$
\min \left\|f^{(n)}\right\| \text {. }
$$

THEOREM 8.2. The unique perfect spline $P^{*}$ involving at most $t-n$ knots in $W_{\infty}^{(n)}(w, d)$ affirmed in Theorem 7.1 provides the unique minimum of (8.3).

Proof. Take $f \in W_{\infty}^{(n)}(\mathbf{w}, \mathbf{d})$ distinct from $P^{*}$. The orthogonality relations 


$$
\int\left(f-P^{*}\right) w_{k}=0, \quad k=1,2, \cdots, t+1,
$$

imply that $f-P^{*}$ actually changes sign at least $t+1$ times. Invoking Rolle's theorem repeatedly we deduce that $f^{(n)}-P^{*(n)}$ changes sign at least $t+1-n$ times. But $P^{*}$ possesses exactly $t-n$ knots so that it follows $\left\|f^{(n)}\right\|>\left\|P^{*(n)}\right\|$ for otherwise we have a contradiction.

With the solution of Problem I at hand we can resolve the following problem.

Problem II. Let $\left\{c_{i}\right\}_{i=0}^{r}$ be given real numbers not all zero. Let $\pi_{t+1}$ designate the class of all real "polynomials" $\pi(x)=\sum_{i=1}^{t+1} a_{i} w_{i}(x)$ (linear combinations of $\left.w_{i}(x)\right)$ and assume $\left\{w_{i}(x)\right\}$ constitutes a Tchebycheff system on [0, 1]. Determine

$$
\sup _{\pi \in \pi_{t+1}}\left[\left|\sum_{i=1}^{t+1} a_{i} c_{i}\right| / \int_{0}^{1} \ln (x) \mid d x\right] .
$$

(For $\pi(x) \equiv 0$, interpret the ratio as zero.) Equivalently consider the class 2 of all polynomials $\pi(x)=\sum_{i=1}^{t+1} a_{i} w_{i}(x) \in \pi_{t+1}$ satisfying the constraint $\sum_{i=1}^{t+1} a_{i} c_{i}$ $=1$ and determine

$$
\min _{\pi \in 2} \int_{0}^{1}|\pi(x)| d x
$$

The equivalence of the two versions follows by multiplication of $\pi(x)$ by a constant since (8.4) is invariant under such scalings. The solution of (8.5) (and so (8.4)) is easily extracted from the resolution of Problem I as will now be demonstrated.

Solution of Problem II. Form

$$
U_{\infty}^{0}=\left\{f \mid f \in W_{\infty}^{(0)}, \int_{0}^{1} w_{i}(x) f(x) d x=c_{i}, i=1,2, \cdots, t+1\right\}
$$

so $U_{\infty}^{0}$ consists of all bounded functions obeying the indicated moment conditions. We obtain

$$
\sum_{i=1}^{t+1} a_{i} c_{i}=\sum_{i=1}^{t+1} a_{i} \int_{0}^{1} w_{i}(x) f(x) d x=\int_{0}^{1} \pi(x) f(x) d x
$$

yielding manifestly the inequality

$$
\left|\sum_{i=1}^{t+1} a_{i} c_{i}\right| \leqslant\|f\|_{\infty} \int_{0}^{1}|\pi(x)| d x,
$$

where $\pi(x) \neq \equiv$ (i.e. $\left\{a_{i}\right\}$ are not all zero). (8.6) implies

$$
\sup _{\pi \in \pi_{t+1}}\left[\left|\sum_{i=1}^{t+1} a_{i} c_{i}\right| / \int_{0}^{1}|\pi(x)| d x\right] \leqslant \inf _{f \in U_{\infty}^{0}}\|f\|_{\infty} .
$$


But $\inf _{f \in U Q}\|f\|_{\infty}$ according to Theorem 8.2 is achieved uniquely by a perfect spline $P^{*}(x)$ with certain knots $0<\xi_{1}<\xi_{2}<\cdots<\xi_{s}<1, s \leqslant t$. Construct $\pi^{*}(x)$ as the polynomial $\Sigma_{i=1}^{t+1} a_{i}^{*} w_{i}(x)$ (unique apart from a multiplicative constant) vanishing precisely at $\left\{\xi_{i}\right\}_{i=1}^{s}$. Thus $P^{*}$ and $\pi^{*}$ change signs together as $x$ traverses $[0,1]$. With this determination of $\pi^{*}$ it follows that equality persists in (8.7). Further scrutiny reveals that the solution is achieved exclusively for the polynomials $a \pi^{*}(x)$ where $a$ is an arbitrary real nonzero constant.

A different approach to the solution of (8.5) is due to Krein [8], (see also Karlin and Studden [7, Chapter 8, §11]). The polynomial $\pi^{*}(x)$ in the special case $c_{t+1}=1, c_{i}=0, i=1, \cdots, t$, and $w_{i}(x)=x^{i-1}, i=1,2, \cdots, t+1$, can be identified (modulo suitable affine transformation) as the Tchebycheff poly. nomial of the second kind.

\section{REFERENCES}

1. S. D. Fisher and J. W. Jerome, Perfect spline solutions to $L^{\infty}$ extremal problems (preprint).

2. G. Glaeser, Prolongement extrema de fonctions différentiables d'une variable, J. Approximation Theory 8 (1973), 249-261.

3. S. Karlin, Total positivity: Vol. 1, Stanford Univ. Press, Stanford, Calif., 1968. MR 37 \#5667.

4. Total positivity, interpolation by splines, and Green's functions of differential operators, J. Approximation Theory 4 (1971), 91-112. MR 43 \#780.

5. - Some variational problems on certain Sobolev spaces and perfect splines, Bull. Amer. Math. Soc. 79 (1973), 124-128.

6. S. Karlin and J. M. Karon, Poised and non-poised Hermite-Birkhoff interpolation, Indiana Univ. Math. J. 21 (1971/72), 1131-1170. MR 47 \#3877.

7. S. Karlin and W. Studden, Tchebycheff systems: With applications in analysis and statistics, Pure and Appl. Math., vol. 15, Interscience, New York, 1966. MR 34 \#4757.

8. M. G. Krein, The ideas of P. L. Čebyšev and A. A. Markov in the theory of limiting values of integrals and their further development, Uspehi Mat. Nauk 6 (1951), 3-120; English transl., Amer. Math. Soc. Transl. (2) 12 (1959), 1-121. MR 13, 445; 22 \#3947a.

9. R. Louboutin, Sur une bonne partition de l'unite. Appeared in Le prolongateur de Whitney. Vol. II; Ed. Glaeser, Univ. of Rennes, 1967.

10. I. J. Schoenberg, The perfect B-splines and a time optimal control problem, Israel J. Math. 8 (1971), 261-275.

11. I. J. Schoenberg and A. Cavaretta, Solution of Landau's problem concerning higher derivatives on the half line, Report No. 1050, M.R.C., University of Wisconsin, Madison, Wis., 1970.

12. V. M. Tihomirov, Best methods of approximation and interpolation of differentiable functions in the space $C_{[-1,1]}$, Mat. Sb. 80 (122) (1969), 290-304 = Math. USSR Sb. 9 (1969), 275-289. MR 41 \#703.

DEPARTMENT OF MATHEMATICS, STANFORD UNIVERSITY, STANFORD, CALIFORNIA 94305 